\title{
Electrostatic interactions in biological DNA-related systems
}

\author{
A. G. Cherstvy* \\ Received 6th December 2010, Accepted 3rd February 2011 \\ DOI: $10.1039 / \mathrm{c0cp02796k}$
}

In this perspective article, we focus on recent developments in the theory of charge effects in biological DNA-related systems. The electrostatic effects on different levels of DNA organization are considered, including the DNA-DNA interactions, DNA complexation with cationic lipid membranes, DNA condensates and DNA-dense cholesteric phases, protein-DNA recognition, DNA wrapping in nucleosomes, and inter-nucleosomal interactions. For these systems, we develop a theoretical framework to describe the physical-chemical mechanisms of structure formation and anticipate some biological consequences. General biophysical principles of DNA compaction in chromatin fibers and DNA spooling inside viral capsids are discussed in the end, with emphasis on electrostatic aspects.

\section{Introduction}

Many constituents of living cells often bear large charges on their surfaces. The list includes nucleic acids, ${ }^{1}$ cellular lipid membranes, ${ }^{2}$ DNA binding ${ }^{3,4}$ and architectural ${ }^{5,6}$ proteins, natural ion channels ${ }^{7}$ and pores, ${ }^{8}$ elements of cytoskeleton networks, ${ }^{9}$ and molecular motors. ${ }^{10}$ ES interactions on the nanoscale often dominate the physical forces acting between these components in the last $1-3 \mathrm{~nm}$ prior to surface-surface contact, governing their spontaneous assembly and longrange spatial ordering. There has been a number of excellent reviews covering the general principles of ES behavior of

Institute of Complex Systems, ICS-2, Forschungszentrum Jülich, 52425 Jülich, Germany.E-mail: a.cherstvy@gmail.com

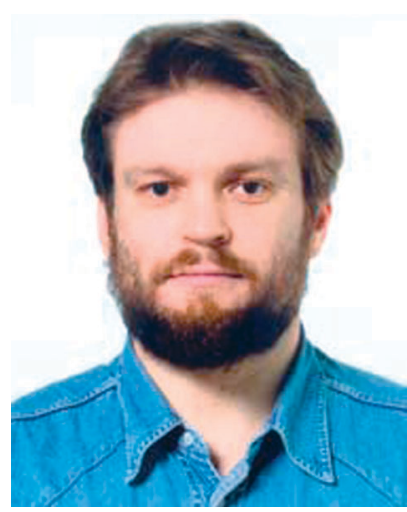

A. G. Cherstvy
Andrey Cherstvy received his Diploma in theoretical physics from Belarus State University in 1998 and PhD in theoretical biophysics from Düsseldorf University in 2002. After two PostDoc terms, at the IFF-2 Institute in the Research Centre Jülich and MPI-PKS in Dresden, he currently holds a research position at the ICS-2 Institute in Jülich (http://www2.fz-juelich.de/iff/ Cherstvy_A), funded by the German Research Foundation, $D F G$. He is the author of more than 25 original papers, with main research interests in theoretical and computational biophysics of bio-molecular assemblies, DNA-protein recognition, and polyelectrolytes. nucleic acids, ${ }^{11,12}$ proteins, ${ }^{13-18}$ lipid membranes, ${ }^{2,19,20}$ and some other bio-soft-matter systems. ${ }^{21}$ Salt- and $\mathrm{pH}$-sensitivity of ES forces provides a useful handle for cells to direct and tune the pathways of many biological processes. Among them are, in particular, the DNA-DNA, ${ }^{11}$ protein-DNA ${ }^{22}$ and protein-protein ES interactions, ${ }^{23}$ DNA compactification into higher-order structures, ${ }^{24,25}$ DNA spooling inside viruses, ${ }^{26}$ actin aggregation into bundles, ${ }^{27}$ RNA folding, ${ }^{12,28}$ and ion translocation through membrane pores. ${ }^{29}$ ES forces modulate the structure and functioning of several sub-cellular supramolecular assemblies ${ }^{30,31}$ and, on a higher level, affect cell-cell interactions in tissues and with substrates. ${ }^{32}$

Over the last several years, ES mechanisms of some DNArelated phenomena mentioned have been developed in our group. General physical principles of the classical PB theory often provide an adequate description of ES properties of molecules in solution and macromolecular complexes. In this perspective, we avoid complicated algebra: all analytical expressions, details of their derivation, and regimes of applicability of the models used can be found in original papers. We rather focus on underlying physical mechanisms, comparing and contrasting the system behavior under different conditions. We often treat ES forces in dense, weakly fluctuating structures and complexes, where entropic effects are often rather weak and can be neglected. When applicable, we also touch on non-ES effects due to e.g., hydration $^{33}$ and electrodynamic ${ }^{34}$ forces. Because of space limitations, we primarily focus on latest ES-motivated developments from other groups, trying to position our research in this context.

Every section below starts with a short introduction to the subject, followed by a presentation of basic theoretical concepts and main results, and supplemented by some perspectives for future developments and possible model improvements. We start with description of ES effects in dense DNA assemblies and condensates. Using the theoretical concepts introduced for a pair of DNA duplexes, the focus is then shifted to 

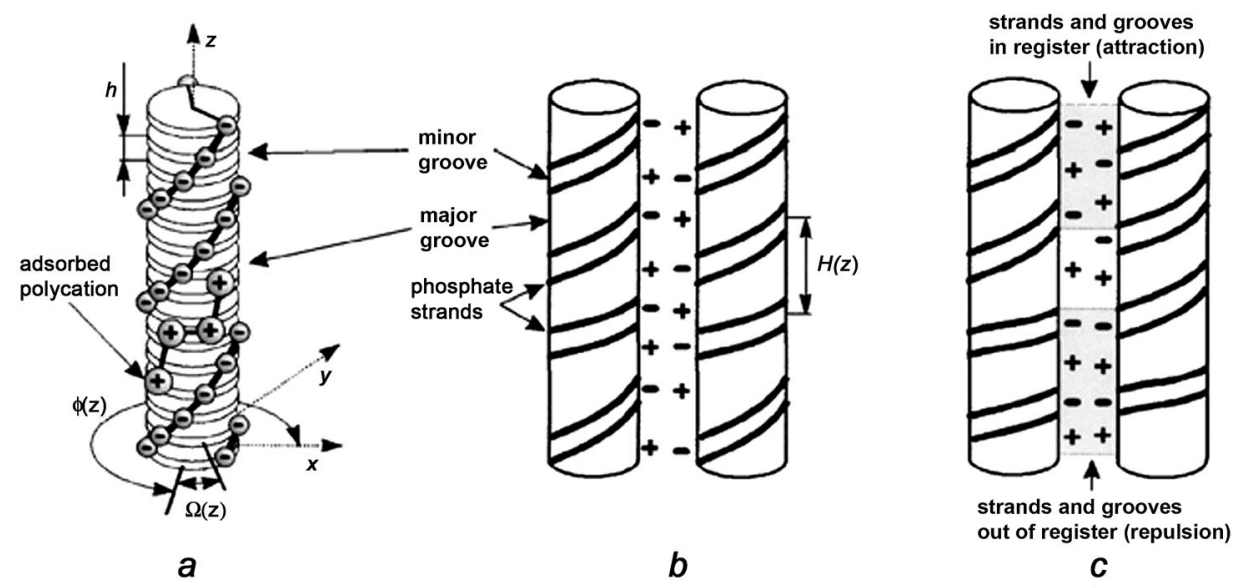

Fig. 1 Schematic model representation of cations-decorated ds-DNA (a) and interacting hom vs. non-hom DNA sequences (b, c). Figure is reprinted from ref. 80, subject to APS-2001 Copyright.

DNA-protein ES interactions, DNA wrapping in NCPs, and protein-mediated DNA looping. The main directions of our future DNA-focused ES research are explained in the end, in particular, the structure of $30 \mathrm{~nm}$ chromatin fibers mediated by inter-nucleosomal interactions, the peculiarities of DNA packing inside viral shells, and some features of self-assembly of viral capsid proteins.

\section{DNA-DNA ES forces: repulsion vs. attraction}

B-DNA is one of the most highly charged biological helices, with one elementary charge $e_{0}$ per $\sim \mathrm{nm}^{2}$ on DNA surface in standard $\mathrm{pH}$ and physiological [salt] conditions. The phosphate negative groups are located on the DNA periphery, forming a duplex with $10-10.5$ bp per helical turn of $H \approx 34 \AA$ and nonhydrated "dry" DNA radius of $a \approx 9 \AA$. More that $\sim 75 \%$ of DNA charge is neutralized by counterions adsorbed onto DNA from solution. The Manning theory of counterion condensation ${ }^{35-37}$ predicts $\theta_{M}=76 \%$ of charge compensation for monovalent $(z=1)$ and $\theta_{M}=92 \%$ for tri-valent $(z=3)$ cations. In the DNA model as a thin long linear PE at vanishing [salt], the neutralization fraction is predicted to be

$$
\theta_{M}=1-1 /(z \xi)
$$

where $\xi$ is the ratio of the Bjerrum length $\left(l_{B} \approx 7.14 \AA\right.$ in water at room temperature) to the axial PE charge-charge separation, $b \approx 1.7 \AA$. Recent translocation experiments of DNA chains through nm-sized solid-state nano-pores enabled measuring the neutralization fractions for DNA, ${ }^{38-40}$ often in good agreement with the Manning theory.

DNA structure offers well-defined sites for counterion binding. Depending on the chemical nature and valence, cations bind preferentially in DNA grooves, on DNA strands, or both, see ref. 11. Spatial distribution and binding equilibrium of adsorbed counterions define their pattern on the DNA surface that, in turn, dictates the features of DNA-DNA ES forces.
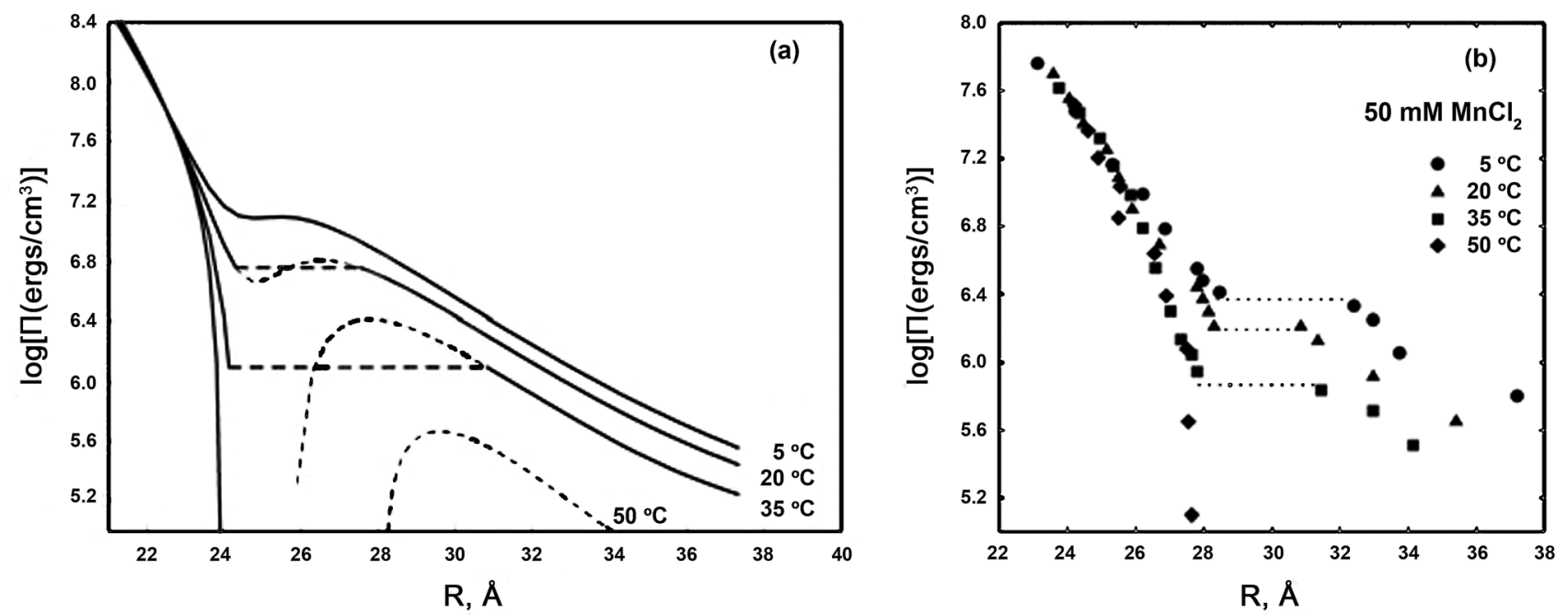

Fig. 2 Theoretically predicted (a) and experimentally measured (b) DNA-DNA forces in dense DNA assembly with $50 \mathrm{mM}^{\circ} \mathrm{MnCl}_{2}$ at a varying $T$. The region of DNA-DNA attraction at $R=28-32 \AA$ in experiments corresponds to a spontaneous collapse of DNA lattice (in this region DNA-DNA energy increases with $R$ ). The case of no azimuthal frustrations on DNA lattice was assumed, i.e., cos $\delta \phi \equiv 0$ for all DNA pairs. Parameters: $\theta=0.85, n_{0}=50 \mathrm{mM}$. Figure is reprinted from ref. 81, subject to ACS-2002 Copyright. 
This pattern of charges also affects the intrinsic DNA helical structure and the particular form DNA adopts in solution. ${ }^{41}$ DNA-DNA ES forces are believed to dominate the intermolecular interactions for parallel DNA duplexes in the last $20 \AA$ prior to surface-to-surface contact, because of still relatively large residual DNA charge density after the counterion condensation.

DNA-DNA hydration force created by overlapping patterns of structured water molecules on DNA surfaces is another possible alternative. ${ }^{42}$ Close similarities in the magnitude and decay length of repulsive forces in the last 1-2 nm prior to contact measured in simple-salt solutions for DNAs, several net-neutral helical polymers ${ }^{43,44}$ and lipid membranes ${ }^{19,45}$ speak in favor of the hydration force picture. Van der Waals attractive interactions, particularly important for interacting of lipid bilayers with large lateral charge mobility and dynamically generated charge fluctuations, seem to have a smaller effect on the forces between DNAs, with their rather static pattern of negative charges. The extreme sensitivity of measured DNA-DNA forces to the nature and valence of added cations, not supposed to affect strongly the close-range hydration forces, ${ }^{19}$ favors however an ES mechanism of DNA-DNA force generation. In particular, the DNA-DNA attraction detected with multi-valent cations can be quantitatively rationalized in the ES models, see below.

The patterns of condensed cations bear some correlations to the helical symmetry of DNA phosphates, forming a lattice of alternating positive-negative charges along the DNA axis, Fig. 1. ES forces between these periodic arrays of charges might turn from repulsion to attraction for well-neutralized DNA duplexes. Attractive DNA-DNA forces have been systematically measured in solutions of some di- and many tri-valent cations at $\approx 1 \mathrm{~nm}$ between DNA surfaces, ${ }^{46-48,55}$ Fig. 2, while purely repulsive ES forces are detected in solutions of monovalent salts. ${ }^{49}$ The list of DNA condensing agents includes many multivalent cations $\left(\right.$ cohex $^{3+},{ }^{50}$ spermine $^{4+}, 51$ spermidine $^{3+52}$, some highly positively charged proteins (poly-Lysine ${ }^{53,54}$ poly-Arginine, ${ }^{42}$ protamines ${ }^{56} \mathrm{H} 1$ histones ${ }^{57}$ ), as well as concentrated solutions of neutral polymers (PEG). The latter are excluded from the DNA phase because of their size, exerting an external osmotic pressure onto the DNA lattice. ${ }^{47}$ Some counterions in this list interact with DNA in natural environments, such as spermidine ${ }^{3+}$ that is present in many bacteria at $1-3 \mathrm{mM}$ concentrations,${ }^{58}$ protamines that are abundant in sperm heads, as well as putrescine ${ }^{2+}$ and spermidine $^{3+}$ that are vital for DNA compaction in some T-even bacteriophages. ${ }^{59}$

A number of theoretical models on different levels of sophistication have been developed in the last two decades to offer physical interpretation of DNA-DNA attraction, including the recent advances. ${ }^{60-63}$ In one group of models, the spatio-temporal correlations of cations originate from the inherent DNA structure and DNA-DNA attraction is possible via a "charge zipper effect". In other models, beyond the PB limit, the correlated fluctuations in counterion density profiles give rise to ES DNA-DNA attraction, ${ }^{64-67}$ even for DNAs modelled as a uniformly charged rods. The period of oscillatory charge density waves on the PE surfaces in these models is largely decoupled from intrinsic DNA charge periodicity.
To save space, we address the reader to a comprehensive recent review ${ }^{11}$ focused primarily on ES forces between DNA duplexes. It provides broad coverage, physical comparison, and analysis of applicability regimes for various models of PE-PE like-charge attraction. DNA-DNA attraction has also been extensively investigated by computer simulations, see e.g. ref. 68-73, in different models for DNA grooved structure, counterion shape and binding specificity, as well as solvent models.

Although the helicity effect on the distribution of ES potential near a single DNA in solution was realized long ago, see e.g. ref. 74 , the exact theory of ES forces between two long parallel double-helical macromolecules has been developed only in 1997 by A. Kornyshev and S. Leikin in a seminal paper. ${ }^{75}$ This elegant, albeit mathematically involved linear PB theory explicitly accounts for DNA helical structure and its low-dielectric hydrophobic core, with permittivity $\varepsilon_{c} \sim 2$. The model assumes the Manning fraction of cations irreversibly adsorbed in DNA grooves or on DNA strands, while the remaining DNA charge is screened by electrolyte ions in the linear, DH manner. DNA ES potential renormalized in this fashion often does not exceed $25 \mathrm{mV}$, rendering the linear $\mathrm{PB}$ model applicable for description of interacting clouds of mobile charges around DNAs.

Both DNA phosphate groups and the condensed cations in the middle of DNA grooves are often modelled as thin continuous helical lines of charges, defining the level of coarse-graining in the theory. Thermal smearing of DNA helical pattern can be incorporated in the model via a Debye-Waller factor ${ }^{11}$ which reduces the magnitude of helical interaction coefficients $a_{1,2}$, see below.

Cations' partitioning between DNA grooves is set in the model by the parameter $0<f<1$, the fraction of cations adsorbed in DNA minor groove. The theory predicts ES attraction between well-neutralized DNAs with the majority of cations adsorbed in the major groove, an effect pioneered in ref. 76. This arrangement of charges facilitates the periodic positive-negative charge alternation along the DNA axis. In physical terms, the attraction emerges from a zipper-like ES matching of phosphate groups of one DNA with the cations adsorbed in a regular fashion in grooves of another DNA. Correlated ES potential barriers generate charge interlocking along the DNA-DNA contact. For DNA-DNA separations of $R=28-35 \AA$ the ES helix-helix attraction overwhelms the image-force and direct DH charge repulsion ${ }^{77}$ that renders net DNA-DNA ES forces attractive. The mathematical apparatus implemented for derivation of ES DNA-DNA forces, ES and chemical details of counterion-DNA binding, as well as the applicability regimes of this mean-field continuum DH-Bjerrum theory are discussed in detail in ref. 11.

Further developments of the theory enabled to incorporate the fine, realistic details of a DNA structure, such as the discrete nature of adsorbed cations ${ }^{78}$ and sequence-specific pattern of twist angles ${ }^{79}$ between the adjacent DNA bps. ${ }^{80}$ The models for interaction- and $T$-mediated rearrangements of condensed cations on DNA surfaces, ${ }^{81}$ torsional flexibility of DNA backbone, ${ }^{82}$ soliton-like DNA twist deformations, ${ }^{83}$ and DNA helical "straightening" in dense DNA crystals ${ }^{84}$ have also been developed. ES forces between non-parallel 
long $^{85}$ and finite-length DNA duplexes ${ }^{86}$ were elucidated and the detailed statistical theory of dense DNA assemblies has been developed. ${ }^{87}$

Many outcomes of the theory are in quantitative agreement with the experimental data available for dense DNA assemblies. These include in particular the decay length of measured DNA-DNA repulsion in simple salt and DNA-DNA attraction at $R=28-32 \AA$ with multivalent cations, see ref. 81 and Fig. 2. Also, the picture of DNA azimuthal frustrations, ${ }^{82,88}$ DNA torsional straightening, ${ }^{89}$ and a reduced positional order in dense DNA lattices ${ }^{90}$ have been rationalized by this theory. Recent theoretical developments unravelled the effect of DNA thermal undulations onto DNA-DNA forces, ${ }^{91,92}$ with a conclusion that helicity-mediated DNA-DNA ES forces might be enhanced in dense DNA columnar assemblies at finite $T$. The effect of binding-unbinding equilibrium of finite-sized cations on intermolecular forces has recently also been clarified in a rigorous self-consistent investigation. ${ }^{93}$ Several important biological implications of the predicted ES duplex-duplex forces were analyzed in the recent perspective article. $^{94}$

DNA-DNA ES interaction energy in electrolyte solution can be approximated as a sum of the first helical interaction harmonics $^{11}$

$$
E(R, L) \approx L\left[a_{0}(R)-a_{1}(R) \cos \delta \phi+a_{2}(R) \cos 2 \delta \phi\right],
$$

where the ES interaction of uniformly charged rods of length $L$ enters in the first term, while helix-specific forces are described by $a_{1,2}>0$ terms. These coefficients decay nearly exponentially with separation $R$, Fig. 3 , and their values depend on the cations' partitioning on DNA and DNA charge compensation fraction $\theta$ as follows ${ }^{76,82}$

$$
\begin{aligned}
a_{0}(R)= & \frac{8 \pi^{2} \bar{\sigma}^{2} a^{2}}{\varepsilon}\left[\frac{(1-\theta)^{2} K_{0}(\kappa R)}{\left[\kappa a K_{1}(\kappa a)\right]^{2}}\right. \\
& \left.-\sum_{n, m=-\infty}^{\infty} \frac{\tilde{f}(n, \theta, f)^{2} K_{n-m}^{2}\left(\kappa_{n} R\right) I_{m}^{\prime}\left(\kappa_{n} a\right)}{\left[\kappa_{n} a K_{n}^{\prime}\left(\kappa_{n} a\right)\right]^{2} K_{m}^{\prime}\left(\kappa_{n} a\right)}\right] \\
a_{m=1,2}(R)= & \frac{16 \pi^{2} \bar{\sigma}^{2} a^{2}}{\varepsilon} \frac{\tilde{f}(m, \theta, f)^{2} K_{0}\left(\kappa_{m} R\right)}{\left[\kappa_{m} a K_{m}^{\prime}\left(\kappa_{m} a\right)\right]^{2}}
\end{aligned}
$$

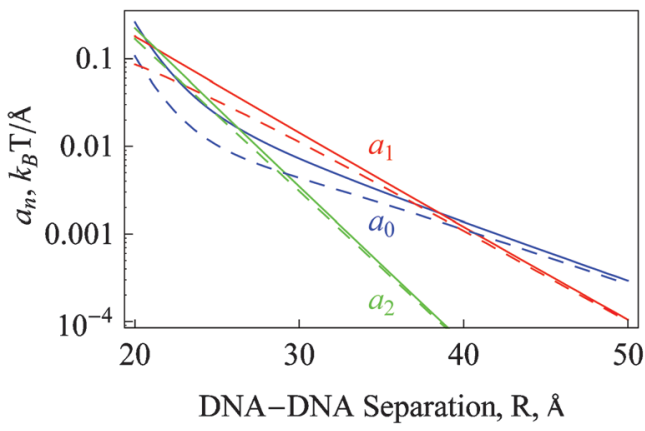

Fig. 3 Dependence of ES helical interaction harmonics for typical DNA model parameters: $\theta=0.8, f=0.3, a=9 \AA, 1 / \kappa=8 \AA$. The solid curves correspond to two DNAs in solution, while the results in dense DNA lattices with the Donnan ionic equilibrium taken into account are plotted as the dashed curves.
Here, the parameter $f$ describes the partitioning of cations between DNA grooves (at $f=0$ all cations are adsorbed in the major groove), $\tilde{f}(n, \theta, f)=f \theta+(-1)^{n}(1-f) \theta-\cos (n \tilde{\phi})$, $\tilde{\phi}_{s} \approx 0.4 \pi$, is the azimuthal half-width of the DNA minor groove, $\bar{\sigma}$ is the bare surface charge density of DNA phosphates, and $K_{n}(x), I_{n}(x), K_{n}{ }^{\prime}(x), I_{n}{ }^{\prime}(x)$ are the modified Bessel functions of the order $n$ and their derivatives.

For ideally-helical DNAs, the interaction energy scales with the DNA length $L$, while for randomly-sequenced fragments a more complicated dependence is anticipated, see section 6 . With the adsorbed cations residing prevalently in the DNA major groove and for large $\theta$ values, the $a_{1}$-term responsible for ES helix-helix attraction grows. The decay lengths for $a_{n=1,2}$ ES contributions,

$$
1 / \kappa_{n}=1 / \sqrt{\kappa^{2}+n^{2}(2 \pi / H)^{2}}
$$

not only contain the DH screening length in $1: 1$ simple-salt solution with [salt] of $n_{0}, \lambda_{D}=1 / \kappa=1 / \sqrt{8 \pi l_{B} n_{0}}$, but also depend on the DNA helical repeat length $H$.

A complicated picture of DNA-DNA azimuthal frustrations in dense hexagonal lattices ${ }^{88}$ emerges from a XY-spin like cos $\delta \phi-\cos 2 \delta \phi$ dependence of DNA-DNA interaction potential on the mutual DNA rotation angle $\delta \phi$. Azimuthal DNA-DNA interactions have to be optimized over 6 DNA molecules on a lattice that inevitably "frustrates" it. ${ }^{95,96}$ The frustrated Potts DNA azimuthal states are often being formed ${ }^{88}$ reminiscent of those for magnetic spin systems.

We mention here that, contrary to the majority of divalent cations including $\mathrm{Ca}^{2+}$ and $\mathrm{Mg}^{2+}, \mathrm{Mn}^{2+}$ and $\mathrm{Cd}^{2+}$ are capable of generating DNA-DNA attraction under osmotic stress of $\mathrm{PEG}^{47}$ and in DNA superhelical plies. ${ }^{97}$ The latter situation imposes an additional confinement onto the DNA strands "jiggling and wiggling" in solution. Both techniques assist to overcome the long-range $\mathrm{DH}$ repulsive branch of the interaction potential and enhance helix-specific DNA-DNA forces screened with a shorter length, $1 / \kappa_{1}$. The short-range branch of DNA-DNA forces originates in the theory from the image-charge ES repulsion of charges on one DNA from the image charges of the same sign induced in the core of the neighboring molecule.

Note that the predicted DNA-DNA short-range repulsion, see Fig. 2, is shifted to somewhat smaller DNA-DNA separations by $3-5 \AA$, as compared to the measured DNA pressuredistance curves. A tentative explanation is that the first DNA tight hydration shell, not included in the model, might effectively increase the physical DNA diameter by the size of the water molecule, preventing direct DNA-DNA contacts at $R \approx 20 \AA$.

The effects of water structuring in hydration shells around the DNA is one of several challenges for this ES theory. Namely, the most interesting features of the intermolecular forces, including the DNA-DNA attraction, emerge at DNA densities when the shells of "structured waters" on DNA surfaces are likely to overlap. Also, a distance-dependent "effective" dielectric permittivity on the length-scales of 1-2 water diameters, ${ }^{13,15,98}$ a modified decay of electric fields close to DNA, finite diameter and specific geometrical form of 

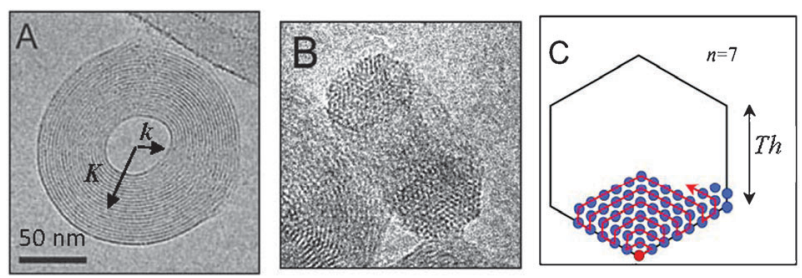

Fig. 4 Cryo-EM images of DNA toroids constructed from 2-3 DNAs $\left(\approx 48.5 \mathrm{kbp}, \lambda\right.$-phage DNA) in $0.2 \mathrm{mM}$ solution of $\operatorname{cohex}^{3+}$ (A, B). The mean $K$ and inner $k$ toroidal radii are indicated. One possible model of defect-free DNA spooling into a torus of generation $n=7$ (C). Image is reprinted from ref. 113, with permission of IOP.

DNA-condensing cations (e.g. linear flexible polyamine chains $v s$. compact cohex ${ }^{3+}$ ), as well as a limited applicability of the PB model, all these factors necessitate the development of more accurate ES theories close to the DNA surface. The effects of solvation ${ }^{99-101}$ around the DNA also require a detailed microscopic treatment of changes in dielectric environments and polarization states upon counterion binding to DNA. These factors also pose lots of complications for quantitative computer simulation-based prediction of DNA-DNA ES forces. Similar obstacles in the description of ES forces on the nano-scale also emerge in the analytical modelling of e.g. DNA-protein, DNA-membrane, and protein-protein complexes, as discussed below.

Calculations of ES forces between other charged helical biomolecules require separate consideration. For instance, attractive RNA-RNA interactions have not been studied systematically, although the counterion condensation on ds-RNAs can be more efficient than on ds-DNAs. ${ }^{102}$ For f-actin bundles, localized counterions follow the underlying charge symmetry to a smaller extent than for DNA, forming rather an organized 1D charge density waves that couple neighboring filaments into a bundle. ${ }^{103}$ Similar to DNA, interaction-induced twist distortions of f-actin filaments have been detected in dense molecular assemblies. ${ }^{9}$ For filamentous viruses, with a multistranded helical distribution of relatively large amino acid blocks, ${ }^{104}$ often some charge "pre-averaging" is required to construct effective charge entities and to derive interaction potentials.

\section{DNA toroidal condensation}

One biological manifestation of cation-mediated DNA-DNA attractive forces is DNA condensation into compact toroidallike structures in vivo (bacteria, viruses, sperm cells) and in vitro. ${ }^{105}$ Some bacteria pack their DNAs into robust toroids to minimize the frequency of ds-DNA breaks. ${ }^{106}$ These radioresistant bacteria retain strongly elevated $\left[\mathrm{Mn}^{2+}\right]$ in their cells to regulate the packaging of chromatin, via likely attractive DNA-DNA interactions. ${ }^{107}$ In mammalian sperm cells, very long DNA is condensed by highly-basic Arg-rich proteins protamines into an assembly of interconnected small toroids, visualized by the AFM technique in ref. 108. DNA compaction inside T5 bacteriophage in the presence of spermine ${ }^{4+}$ also exhibits toroidal-like arrangements for a part of the DNA spool, that likely optimize the energetics of DNA packing. ${ }^{109}$
In vitro, DNA toroids with cohex $^{3+}$ visualized by the cryo-EM reveal a spool-like DNA organization into a torus with $\sim 50 \mathrm{~nm}$ outer and $\sim 15 \mathrm{~nm}$ inner radius, ${ }^{110}$ with nearly hexagonal DNA local lattice order, Fig. 4. In case when several DNAs comprise a toroid, the most frequently encountered condensates contain an optimal number of DNA molecules. Often, almost hexagonal toroidal cross-sections are detected, with completely filled outer DNA shells that correspond to the most stable aggregates. DNA-DNA separations in toroids are often $R \approx 28 \AA$, being in the range of DNA-DNA attraction as measured by the osmotic stress and as predicted by the theory of DNA-DNA ES interactions, see Fig. 2.

Note here that in another example of dense DNA assembly, in 3D DNA origami structures, very small DNA-DNA separations of $R \approx 22-25 \AA$ are typically realized. ${ }^{111,112} \mathrm{~A}$ successful assembly process requires $\sim 10-20 \mathrm{mM}$ of divalent $\mathrm{MgCl}_{2}$ salt, that is likely to reduce the ES repulsion of DNA strands during the assembly process driven by the chemical energy of the association of complementary ss-DNA fragments.

Utilizing these facts, we constructed a simple model of DNA toroid growth by generations. ${ }^{113}$ Due to a finite DNA bending persistence length $l_{\mathrm{p}},{ }^{114,115}$ DNA toroidal structures are often preferred over rod-like or spherical DNA condensates. ${ }^{116}$ During the first stage of compaction, the initial DNA circular loop is thermally nucleated and stabilized, with the curvature radii $\sim l_{\mathrm{p}}$. Toroids' growth in the model is controlled by an interplay of ES DNA-DNA attractive forces and DNA elastic deformation energy. ${ }^{113}$

As the toroidal cross-section grows, the fraction of missing DNA-DNA attractive "bonds" on the toroid surface progressively decreases. This improves the ES attractive energy gain per unit length of DNA compacted, approaching the value for a DNA columnar phase, where the pair DNA-DNA interaction energy is enhanced 3 times due to 6 neighboring DNAs. On the other hand, as toroidal cross-section grows, the DNA wrapping near the inner "hole" is accompanied by a higher bending energy. The optimal toroidal radius $K$ and thickness $T h$ follow the scaling relations $K \propto\left|E_{0}\right|^{-2 / 5} L^{1 / 5} l_{p}^{2 / 5}$ and $T h \propto\left|E_{0}\right|^{+1 / 5} L^{2 / 5} l_{p}{ }^{1 / 5},{ }^{113}$ as a function of the DNA-DNA attraction strength at an optimal DNA density $E_{0}=E\left(R_{\text {opt }}\right)$ and for a DNA of length $L$. According to the DNA-DNA ES theory, ${ }^{11}$ see eqn (2), in the presence of DNA-condensing cations the DNA-DNA cohesive energy can reach $E_{0}=$ -(0.01-0.1) $k_{\mathrm{B}} T$ per bp along the DNA-DNA contact. It plays the role of surface tension controlling the toroidal dimensions, see Fig. 5. The model shows that as the DNA persistence length decreases and DNA-DNA attraction increases, the toroids become "fat": their mean radius is reduced and the thickness grows.

Several models of DNA toroidal condensation for nonhexagonal and non-circular cross-sections were employed in the literature. ${ }^{117-121}$ We also want to mention that, although locally the lattice of wrapped DNA preserves the hexagonal symmetry to maximize the attraction energy gain from intermolecular contacts, the path taken by a continuous long DNA strand upon wrapping in toroids is still under debate. ${ }^{122,123} \mathrm{~A}$ similar question emerges for DNA packing inside the viral capsids, see section 12 . 


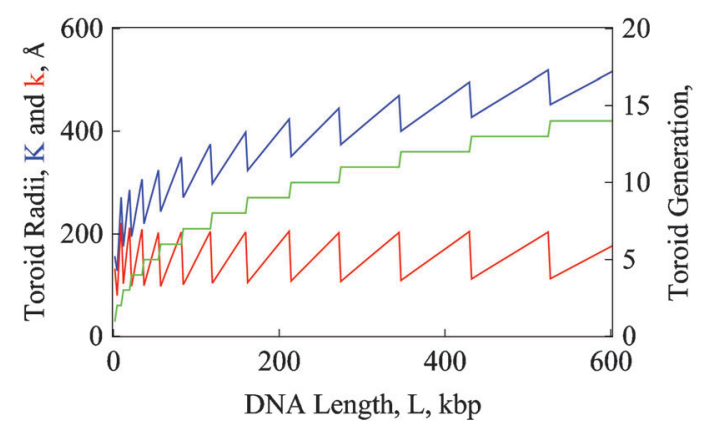

Fig. 5 Mean $K$ and inner $k$ radii of DNA toroids of generation $n$, as obtained at relatively strong DNA-DNA attraction of $E_{0}=-0.05$ $k_{\mathrm{B}} T / \AA \AA^{113}$ The saw-tooth variation of toroidal dimensions is due to the growth-by-generation model implemented.

Recent single-molecule optical tweezers manipulation experiments have enabled to decipher the physical mechanisms behind the toroidal stability and DNA condensation dynamics. ${ }^{124}$ In particular, a step-wise unwrapping of DNA from toroids by the applied force has been detected, corresponding to multiple DNA loops released from the condensate. The number of turns released was shown to be a function of the applied tension of 1-10 $\mathrm{pN}$ and salt-mediated DNA-DNA attraction. Theoretical mechanical ${ }^{125}$ and statistical ${ }^{126}$ models of forceinduced DNA unwrapping from DNA toroidal aggregates have also been developed in recent years.

One intriguing perspective is DNA globular and toroidal condensation on positively charged surfaces. Some mechanical studies of toroidal compression between wetting and nonwetting surfaces have been performed in the past, ${ }^{127}$ without accounting however for ES effects inside condensates and on condensate-surface interphase. DNA condensation in 2D on positively charged surfaces ${ }^{128}$ and membranes is expected to follow a different pathway and results in different final morphologies, as compared to 3D DNA aggregation.

Recently, a coil-globule DNA transition on unsupported CL membranes has been reported. ${ }^{129}$ The DNA globules of $\sim 0.1-0.4 \mu \mathrm{m}$ in size emerge in $1: 1$ salt solely due to $\mathrm{CL}$ charges mobile on 2D membrane. They serve as counterions for DNA, compensating its charge along the DNA-membrane contact. The precise morphology of the condensates could not be resolved, ${ }^{129}$ but the hydrodynamic radii of DNA globules were dramatically reduced with the increasing fraction of $\mathrm{CL}$ in the membrane. One can suggest that CL positive patches get bound to the deposited DNA, progressively wrapping and compacting DNA coil into a globule. The membrane deformations required for this process are vital, because supported membranes with the same lipid composition do not feature such coil-globule DNA compaction. ${ }^{130}$ Mixing and rearrangement of membrane lipids should also contribute, similar to lipid charge adjustment in DNA-(CL membranes) complexes reviewed in the next section.

\section{DNA complexes with lipid membranes}

The self-assembly of CL membranes with oppositely charged bio-macromolecules has recently been studied experimentally for DNA, ${ }^{131}$ f-actin, ${ }^{132}$ microtubules, ${ }^{133}$ and some filamentous viruses. ${ }^{134}$ Dense DNA-CL-membrane assemblies are promising non-viral transfection vectors for gene therapy applications, ${ }^{135}$ targeting nowadays some types of cancer. ${ }^{136}$

The surface charge density on positively charged CL membranes, $+0.3-1 e_{0} / \mathrm{nm}^{2}$, is often comparable to that of negatively charged DNA and ES forces dominate their complexation into different phases. Depending on the membrane charge density, flexibility, and lipid composition, dense well-ordered lamellar $L_{\alpha}{ }^{c 131}$ and inverted-hexagonal $H_{I I}{ }^{c}{ }^{137}$ phases are commonly observed, Fig. 6 . The latter are preferred for artificially soft or intrinsically pre-curved membranes, when a tight wrapping of lipid charged heads around the DNA molecules takes place on a lattice. For the DNA-membrane lamellar phase, ordered DNA layers alternate with CL membranes, while for f-actin, due to a mismatch in the charge densities, the unit cell of the lamellar stack consists of two negative f-actin layers on both sides of a positively charged CL-membrane.

For DNA-CL complexes, the most stable assemblies often occur at the isoelectric point of exact charge matching of the DNA and CL membrane. ${ }^{138}$ The process is accompanied by an almost complete release of condensed counterions from the DNA and membrane surfaces. ${ }^{139}$ Concomitantly, the translational entropy of "evaporated" counterions is maximized. The DNA-DNA separations measured in DNA-CL complexes are in the range $25 \AA<R<60 \AA$ and in simple salt solution they often fit the picture of counterion-free assemblies.
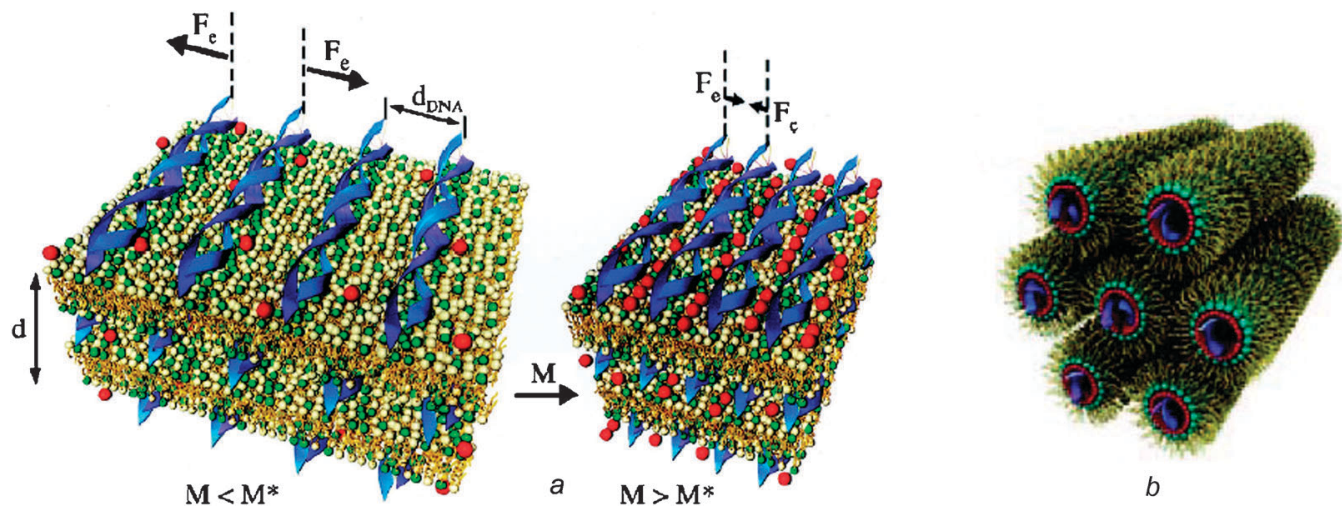

Fig. 6 Schematics of 2D DNA condensation in the lamellar DNA-CL-membrane phase with divalent cations (a). Inverted hexagonal $H_{I I}{ }^{c}$ phase of DNA-(CL membrane) complexation (b). Part (a) of the figure is reprinted from ref. 156, copyright 2000-NAS, USA. 
The ES stabilization mechanism of DNA-membrane complexes based on numerical solutions of non-linear PB equation was established a decade ago with non-fluctuating rod-like DNAs ${ }^{140-143}$ and refined for more realistic setups in recent coarse-grained computer simulations. ${ }^{144-148}$ For the lamellar complexes, particular attention has been paid to ES-driven DNA-mediated adjustment of the CL charge density profile $^{149}$ and membrane undulations ${ }^{150}$ that might help to improve DNA-membrane charge matching.

Recently, we developed a similar ES model based on the exact solutions of linear PB theory, with the dielectric boundaries (DNA-solvent and membrane-water) and DNA helicity taken explicitly into account. ${ }^{151}$ Within this approach, both for $L_{\alpha}{ }^{c}$ phase with planar membranes and for $H_{I I}{ }^{c}$ phase with membranes wrapped around the DNA, the distribution of ES potential in the electrolyte has been calculated. We restored the variation of the complex ES energy as a function of DNA lattice density and CL fraction on the membrane. Both appear to exhibit non-monotonic behavior, in good agreement with numerical results of the nonlinear PB model. ${ }^{141}$ The energy minimum computed from the model roughly corresponds to electro-neutral assemblies. ${ }^{151}$ For the lamellar phase, the energy well near the minimum describes the ES compressibility of the DNA lattice. The scaling law for the compressibility modulus obtained, $B_{\text {comp }} \propto 1 / R^{2}$, agrees well with experimental data available. ${ }^{152,153}$ For $L_{\alpha}{ }^{c}$ phase, the ES energy of DNA-induced undulatory membrane deformations can be included in an advanced model.

The laws of DNA-DNA ES interactions along and across the CL membranes were also examined in the model. ${ }^{151}$ For instance, for two thin rods on a "salty" interface with mobile charges $^{154}$ one can predict a power-law decay of ES interactions, in contrast to a nearly exponential decay of rod-rod ES screening in 3D, see Fig. 7. The confinement of electrolyte between the adjacent membrane layers ${ }^{155}$ also modifies the law of screening for charges interacting along the membrane. Namely, from the exponential decay of the ES potential of a point charge at small distances $d$, it turns into $1 / \mathrm{d}^{3}$ power-law decay at large distances, when the electrolyte in inter-membrane space is rendered quasi-2D. ES interactions across a lowdielectric membrane are also renormalized in a nontrivial fashion. ${ }^{151}$ All these features affect the properties of DNA transversal and longitudinal correlations in DNA-CL lamellar phases as measured in ref. 153.

The theory of DNA-DNA duplex-duplex ES interactions also enables to rationalize ${ }^{151}$ the DNA-DNA separations

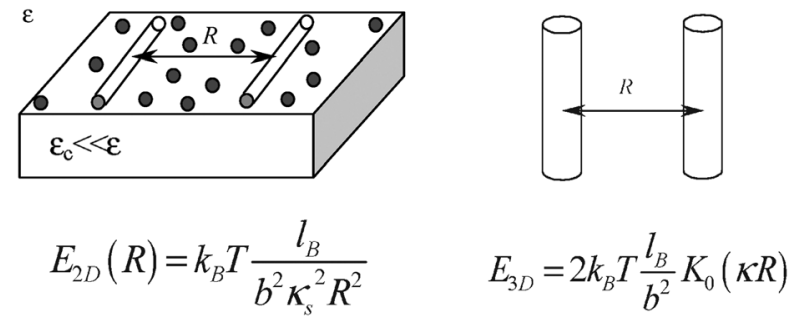

Fig. 7 Energy density of rod-rod ES repulsions along a salty membrane with the inverse Debye length $1 / \kappa_{s}$ and in $3 \mathrm{D}$ electrolyte. measured in $L_{\alpha}{ }^{c}$ phases in the presence of some divalent cations $\left(\mathrm{Mg}^{2+}, \mathrm{Co}^{2+}\right.$, and $\left.\mathrm{Ca}^{2+}\right)$, that are capable of triggering DNA condensation in this 2D DNA-membrane system $^{156}$ but not in $3 \mathrm{D}$ DNA solution. It has been observed that at a critical concentration of divalent salt of $\sim 20-60 \mathrm{mM}$ the DNA-DNA separations drop abruptly from $\approx 45 \AA$ for nearly electro-neutral complexes down to "universal" compaction density with $R \approx 29 \AA$. Also for multivalent spermine ${ }^{3+}$ and spermidine $^{4+}$ cations, the DNA-DNA separations in DNA-CL phase are close to those in 3D lipid-free DNA condensates $^{157}$ and agree with the predictions of the DNADNA ES interactions theory, ${ }^{11,81}$ see Fig. 8. One can conclude that the 2D geometry of DNA-CL $L_{\alpha}{ }^{c}$ phase facilitates DNA-DNA ES counterion-mediated attraction, making the attraction possible even with divalent cations and at a lower DNA charge neutralization fractions, measured to be $\theta \approx 0.63<\theta_{M \cdot}{ }^{156}$

A perspective for future research is to enrich the physical understanding of DNA release from sub- $\mu$ m sized DNA-CL complexes and their translocation into cell cytoplasm across a negatively charged cellular membrane. ${ }^{136,158}$ Both processes are necessary for efficient gene delivery, with the transfection efficiency ${ }^{159}$ of DNA-CL complexes still remaining low as compared to viral-based gene carriers. ${ }^{136}$ It is known for instance that spermine ${ }^{3+}$ and spermidine ${ }^{4+}$ not only condense DNAs in DNA-CL complexes, but can also trigger DNA release from the complexes via DNA condensation into dense aggregates in solution. Another pathway for DNA release is to design the lipid membranes being unstable in particular cellular cytoplasm environments (e.g. via addition of special, "helper" lipids).

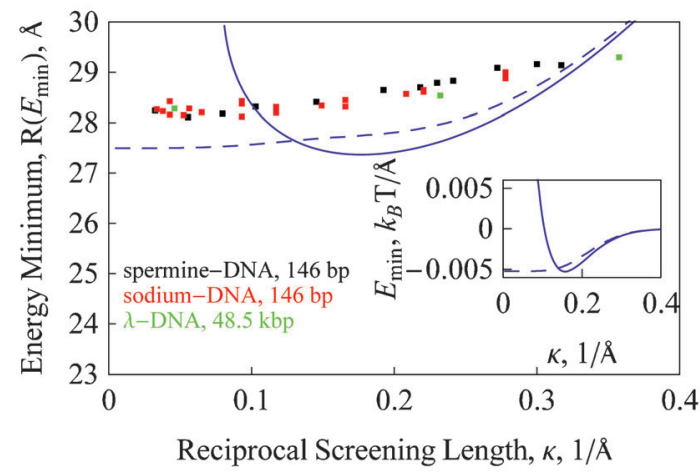

Fig. 8 Optimal DNA-DNA separations as measured in dense DNA precipitates with spermine ${ }^{4+}$ experimentally ${ }^{157}$ (dots) and as predicted theoretically ${ }^{151}$ based on the theory of DNA-DNA ES interactions. ${ }^{76}$ The theory curves with the Donnan saturation (dashed curve, see next section for details) are more realistic, while the solid curves are calculated for unchanged external salt levels also inside the DNA lattice. The amount of added multivalent cations varied in the experiments was assumed in the model to contribute only to a charge in screening: no effects of [spermine] on DNA charge fraction $(1-\theta)$ and of competitive adsorption of multi- $v s$. monovalent cations on DNA were taken into account. The inset depicts the predicted ES DNA-DNA energy in the local energy minimum. Parameters: $\theta=80,70 \%$ of adsorbed cations reside in DNA major groove that gives $f=0.3$. I thank E. Raspaud for providing the experimental data. 


\section{DNA cholesteric phases}

DNA chirality on the nano-scale ${ }^{160}$ manifests itself via DNADNA interactions in the formation of twisted, $\sim 160-350 \mathrm{mg} \mathrm{ml}^{-1}$ dense LC DNA phases on the micro-scale. ${ }^{161-166}$ Cholesteric phases also emerge upon assembly of other bio-helices, e.g., collagen fibers and filamentous viruses ${ }^{167,168}$ and guanosine. ${ }^{169}$ Some polypeptides also feature LC phases. Nature uses the ability of DNA to form chiral phases to pack the genomes in some bacteriophages, ${ }^{170}$ bacteria, ${ }^{171}$ and in the sperm of many vertebrates. $^{172}$

Typically, left-handed DNA cholesteric phases are detected, with the cholesteric pitch of $P \sim 1-4 \mu \mathrm{m}$ for $\sim 150 \mathrm{bp}$ long DNA fragments obtained from NCPs. ${ }^{173}$ The pitch dependences on [salt], ambient temperature $T$, DNA lattice density, and external osmotic stress are however pretty complex functions. For example, the pitch $P$ decreases at higher $[\mathrm{NaCl}]$ in the range $0.2-1 \mathrm{M} .{ }^{173}$ It reaches $P \sim 20 \mu \mathrm{m}$ values for DNA phases with some multivalent cations ${ }^{174}$ and can be reversed by addition of short positively charged polymers such as poly-lysine and chitosan.

Several theories of DNA cholesteric ordering have been developed based on the helical nature of the DNA charge pattern ${ }^{175,176}$ in order to rationalize these and other features in the system behavior. Some geometrical models imply that the right-handed pitch is favored by DNA-DNA steric hindrance, ${ }^{177}$ while left-handed cholesteric phases should originate from ES interactions. ${ }^{104,178}$ Other, purely ES models treat DNA charge helicity explicitly and predict right-handed twist direction to be favored for two right-handed DNAs in close contact. ${ }^{86}$ Such twisting direction ensures (in the contact region of skewed DNAs) a more parallel and thus more ES-favorable arrangement of negative phosphate strands of one partially neutralized DNA with the "strands" of adsorbed counterions on a neighboring DNA. ${ }^{11}$ This causes however a right-handed twist in DNA cholesterics, contrary to the majority of experimental observations.

Based on the theory of ES interactions of two skewed DNAs, ${ }^{85}$ we have examined the ES-stability of DNA cholesteric phases via calculating the strength of DNA-DNA azimuthal correlations, ${ }^{179}$ see Fig. 9. The DNA triad model was implemented, in the ground-state, with no fluctuations, and with a perfect DNA azimuthal register on a lattice. ${ }^{86}$ The theory predicts a non-monotonic pitch dependence on DNA density for $\approx 150$ bp DNA fragments, in agreement with experimental data. ${ }^{173}$ Also, the range of DNA densities of $R=35-45 \AA$ predicted is often close to the measured stability domains of DNA cholesterics, Fig. 10. This indicates that longer-range ES forces, rather than short-range steric hindrance of grooved DNA surfaces, are responsible for DNA LC ordering.

Strong DNA-DNA azimuthal correlations are essential for the formation of DNA cholesterics. The ES model developed predicts that these correlations are reduced both at small and large DNA lattice densities. In the first situation, it happens due to a natural decay of DNA-DNA ES interactions, while in the small- $R$ region the inherent azimuthal frustrations of DNA-DNA interaction potential destroy the DNA orientational order.

The existing ES theory of DNA cholesterics ${ }^{86}$ has been modified to incorporate the Donnan electrochemical equilibrium of

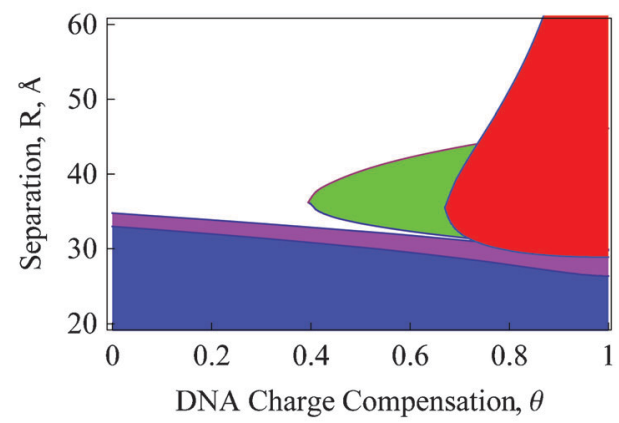

Fig. 9 Stability domain of DNA cholesterics. Azimuthal correlations of $\approx 150$ bp DNA fragments are strong inside the green domain, as predicted by the ES DNA-DNA interaction theory ${ }^{86}$ with the Donnan equilibrium. ${ }^{179}$ The energy of azimuthal DNA rotation on a hexagonal lattice exceeds $k_{\mathrm{B}} T$ in the green region. The $\mathrm{LC}$ twist elastic constant is $K_{22}<0$ inside the red domain, the DNA azimuthal rigidity constant is $k_{\phi}<0$ for magenta and blue domains (all these regions are non-physical). Parameters: $\lambda_{D}=7 \AA, f=0.3$.

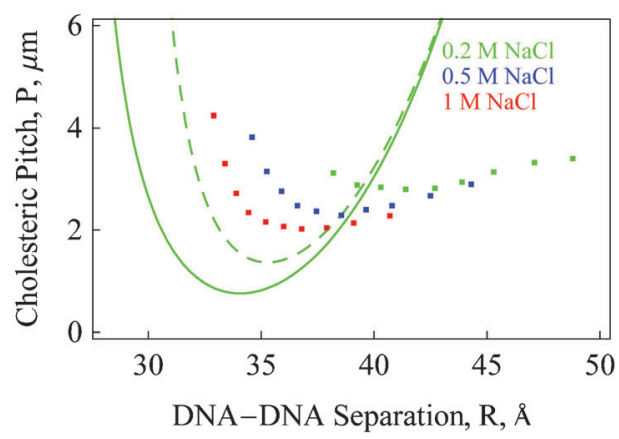

Fig. 10 DNA cholesteric pitch, as calculated with (dashed) and without (solid curve) the Donnan equilibrium on the DNA lattice. ${ }^{179}$ The DNA assembly is pressurized externally to retain a proper [DNA]. The experimental points for DNA cholesteric phases of $146 \mathrm{bp}$ fragments are taken from Fig. 5a of ref. 173. Parameters: $f=0.3$, $\theta=0.65$, and $\lambda_{D}=7 \AA$ that corresponds to $\sim 0.2 \mathrm{M}$ of $\mathrm{NaCl}$.

ions ${ }^{180}$ in DNA lattices. It appears to be particularly important at low [electrolyte], as follows from the equation for renormalized screening length inside a DNA assembly ${ }^{179}$

$$
\lambda_{D}^{\text {Don }} \approx \lambda_{D}\left[1+\left(\frac{4 l_{B} \lambda_{D}^{2}(1-\theta)}{b\left(R^{2} \sqrt{3} /(2 \pi)-a^{2}\right)}\right)^{2}\right]^{-1 / 4} .
$$

Thus, in dense electro-neutral DNA assemblies the DNA lattice density itself dictates the ionic conditions between DNAs, see Fig. 11.

Despite the good agreement for the pitch value, the direction of winding of DNA cholesteric layers and shift of stability domains at different [salt], see Fig. 10, cannot be rationalized by this ES theory in the current form. A right-handed pitch is predicted at relevant DNA densities of $R \sim 35-45 \AA$, with a possible change to left-handedness at very dense DNA packing or for special counterion patterns on the DNA. ${ }^{175}$ In the model of dense LC phases with thermally undulating rather than straight DNAs, a right-to-left pitch inversion might emerge from an enhanced contribution of ES image forces. 


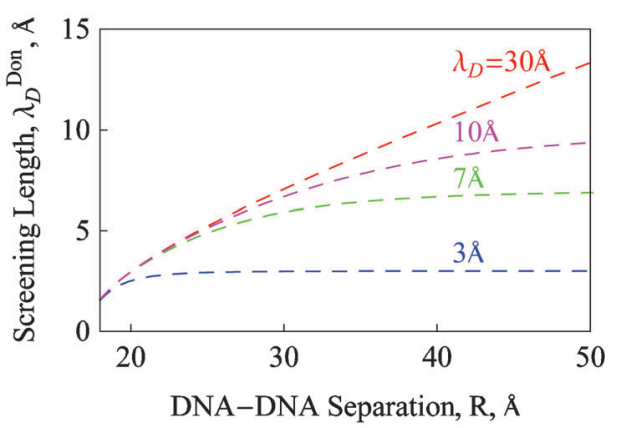

Fig. 11 Effective screening length in a dense DNA assembly for different [salt] in the bulk solution, as calculated within the cell model according to eqn (5).

The latter can favor the opposite sense of DNA-DNA crossing, compared to the direct duplex-duplex ES forces. This conjecture however requires a detailed future analysis. ${ }^{181}$

A major breakthrough in understanding the LC DNA phases has been published recently. ${ }^{182}$ Namely, an inversion of the cholesteric handedness of dense LC DNA phases of 6-20 bp short DNA fragments was revealed as possible. Subtle changes in the DNA sequence and DNA fragment length were shown to be capable to trigger this inversion. These short DNA fragments stack on each other forming elongated DNAs with a sequence-specific 3D structure. ${ }^{183-185}$ For the DNA stacking method that produces more regular helical DNA strands, predominantly left-handed LC phases were observed, similar to those for $\approx 150 \mathrm{bp}$ fragments and longer DNAs. Righthanded DNA cholesterics were observed for short sequences and more azimuthally flexible stacking connections of these nano-DNA rods.

The analysis of data (for a variety of stacked DNA oligomers, at different [DNA] controlled by sample dehydration) enabled the authors to reconcile the results in terms of a single physical parameter. ${ }^{182}$ Namely, for DNA lattices with isotropicnematic phase transition below $\approx 620 \mathrm{mg} \mathrm{ml}^{-1}$, the lefthanded DNA cholesterics are being formed. DNA sequences that experience this transition at larger [DNA] give rise to right-handed DNA LC phases. Thus, for DNA-DNA distances of $R \leq 32 \AA$ the right-handed DNA-DNA crossings seem to be favored $^{186}$ and the LC twist is right-handed for the majority of DNA sequences shorter than 14 bp. ${ }^{182}$

Also, a general tendency has been detected ${ }^{182}$ that shorter DNA oligomers form cholesterics with a shorter, sub- $\mu \mathrm{m}$ pitch. This fact is consistent with the ES theory ${ }^{86}$ being also similar to the $P(L)$ variation for longer DNA molecules. ${ }^{187} \mathrm{~A}$ pitch of $\sim 0.3 \mu \mathrm{m}$ was detected, much shorter than 2-4 $\mu$ m measured for $\sim 150 \mathrm{bp}$ fragments.

The systematic analysis of [salt] and $T$-dependence revealed also several unexplained features. Some sequences exhibit for example a pitch reversal at $[\mathrm{DNA}] \approx 620 \mathrm{~m} \mathrm{ml}^{-1}$. Also, for the majority of DNA oligomers the pitch increases with $T$, indicating an unwinding of cholesteric structures, regardless of its handedness. For many sequences, the pitch was almost insensitive to [salt], contrary to a strong $P([$ salt] $)$ dependence for $\approx 150 \mathrm{bp}$ DNAs measured in ref. 173. All in all, this detailed investigation of twisted phases of nano-DNAs ${ }^{182}$ enriched enormously the widely accepted view of "left-handed-only" DNA cholesterics, challenging future theoretical modelling of DNA twisted phases based on the ES theories of sequence-specific DNA-DNA interactions.

\section{DNA-DNA ES sequence recognition}

So far, the ES forces between ideally-helical parallel or skewed DNA duplexes have been described. The locality of intermolecular ES potential gives also rise to the same strength of ES interactions for DNA fragments hom in sequence but not ideally-helical. Below, the sequence effects on DNA-DNA ES forces are overviewed. To save space, we touch on several subjects only, referring the reader to an excellent recent perspective article $^{94}$ that covers all aspects of the ES theory of DNA-DNA recognition and also suggests biological phenomena where it is of potential importance. One immediate application of the theory is to provide a physical rationale ${ }^{188,189}$ for recognition and pairing of hom genes on genomic ds-DNA molecules ${ }^{190-192}$ during the cell division.

The physical mechanism of ES DNA-DNA sequence recognition has been pioneered in ref. 80 for parallel torsionally rigid DNAs and later extended for DNAs with a realistic value of torsional rigidity in ref. 82. The ES recognition emerges in the model solely due to the inherent bp-specific non-idealities of the DNA helical structure, as extracted from a detailed statistical analysis of structural data on DNA-DNA and DNA-protein crystals. ${ }^{193,194}$ In particular, the DNA bp twist angles are known to exhibit strong variations in DNA-protein crystals $^{79,195}$ fluctuating in the range of $28-40^{\circ}$ that is equivalent to $\approx 36 \pm 5^{\circ}$ deviations.

In the theory, these variations decay along randomlysequenced DNAs with the DNA helical coherence length that is $\lambda_{c}=H /\left(10 \Delta \Omega^{2}\right) \approx 45 \mathrm{~nm}$ at a typical value $\Delta \Omega \approx 5^{\circ}$. These DNA non-idealities strongly affect DNA-DNA ES forces. A finite DNA twist persistence length $l_{t w} \approx 75 \mathrm{~nm}$ allows some interaction-induced DNA torsional adjustments to take place. They restore to some extent the helical register along sequenceunrelated DNA fragments and render the DNA-DNA ES attraction possible again for well-neutralized duplexes. ${ }^{82}$

For torsionally-rigid DNA fragments of length $L$, with the condition of azimuthally free ends, the ES recognition energy in the leading $a_{1}$-approximation is: ${ }^{80,196}$

$$
\Delta E(L) \approx a_{1} \lambda_{c}\left[L / \lambda_{c}+2 e^{-L /\left(2 \lambda_{c}\right)}-2\right] .
$$

In another limit of torsionally adaptable, very soft DNA duplexes, the recognition energy is also described by a simple formula ${ }^{196}$

$$
\Delta E(L) \approx a_{1} \frac{\lambda_{t}}{2 \lambda_{c}}\left[L-\frac{\lambda_{t}}{2}\left(1-e^{-2 L / \lambda_{t}}\right)\right],
$$

where $\lambda_{t}(R)=\sqrt{C /\left[2 a_{1}(R)\right]}$ is the $R$-dependent DNA torsional adaptation length. For the standard DNA twist modulus of $C=750 k_{\mathrm{B}} T \AA$, the exact analytical expressions for $\Delta E(L)$ are however quite cumbersome. ${ }^{82}$ Roughly, at relevant parameters, the theory predicts that two DNA fragments with unrelated sequences attract each other nearly twice as weak as two hom DNA sequences, see Fig. 12a. 

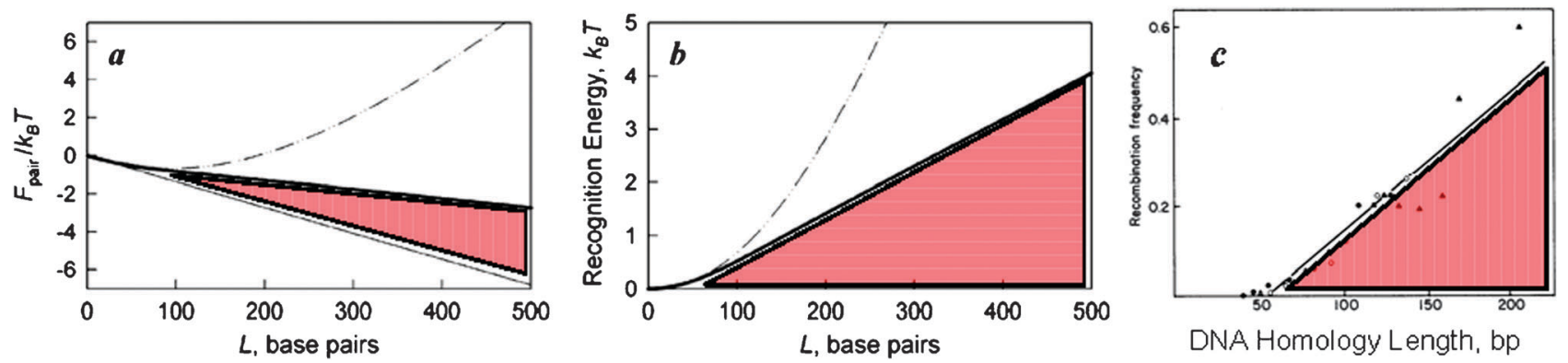

Fig. 12 (a) Theoretical results for the ES interaction energy in a pair of hom DNAs (thin curve), randomly-sequenced torsionally rigid DNA fragments (dot-dashed curve), and randomly-sequenced DNA fragments with realistic twist rigidity $C$ (solid curve). All are plotted at $R=30 \AA$ between the DNA axes. (b) The corresponding DNA-DNA ES recognition energy. Parameters: $\lambda_{D}=7 \AA, \theta=0.8, f=0.3$, and $l_{t w} \approx 750 \AA$. (c) Measured frequency of hom recombination events in the T4 phage. ${ }^{206}$ It exhibits a minimal length of DNA homology $\approx 50$ bp necessary for recombination to start and a linear growth of recombination frequency with DNA homology length. The latter resembles a linear growth of ES DNA-DNA recognition energy for long sequences, as illustrated in part (b). The images are reprinted from ref. 82 with permission of ACS and from ref. 206 with permission of Elsevier.

The ES recognition energy of DNA hom sequences grows linearly with the DNA length in contact, ${ }^{82}$ resembling thereby some properties of DNA hom recombination monitored in vitro in the absence of specific DNA-pairing proteins, see Fig. 12c. ES recognition energy exceeds several $k_{\mathrm{B}} T$ for closely aligned DNA fragments of $\sim 200-500$ bp in length. This energy is large enough to ensure a stable pairing of hom DNAs at ambient temperatures. It might also be sufficient to trigger un-pairing of two DNA single strands, required for initial steps of hom recombination.

When pulling two parallel DNA molecules with hom bp domains in them, the ES recognition energy well emerging near the homology locus has recently been theoretically computed in ref. 188. For very closely juxtaposed DNAs at $R=30 \AA$, recognition energies of up to $5-10 k_{\mathrm{B}} T$ and pinning forces of DNA hom fragments near the bottom of the recognition well $\sim 2 \mathrm{pN}$ have been predicted for typical DNA parameters, see Fig. 13.

Sequence-specific DNA recognition and pairing between intact duplexes was indeed observed experimentally for NCPfree yeast hom DNA chromosomal loci, in the absence of any recA-family proteins. ${ }^{197,198}$ It was attributed to some transient sequence-specific DNA-DNA forces, capable of initiating and maintaining the proximity of hom DNA fragments at separations as large as $\sim 100-300 \mathrm{~nm}$.

Recently, several experimental techniques were utilized to elucidate the properties of DNA-DNA hom pairing in denser arrangements. ${ }^{199-201}$ In one study on dense DNA cholesteric spherullites, the segregation of $\sim 300$ bp DNA fragments with identical bp sequences into separate LC populations has been clearly monitored. ${ }^{200}$ This was a first proof of direct DNA-DNA recognition, based exclusively on DNA bp-sequence information. The effect was attributed to more favorable interactions between DNA hom fragments as compared to non-related ones. ES DNA-DNA bp-specific forces, as predicted by the theory, ${ }^{188}$ are likely to be responsible for the observed segregation of hom sequences at these relatively high [DNA] corresponding to $R=32-40 \AA$.

In another study, single-molecule magnetic tweezer measurements revealed an efficient sequence-specific pairing of $\lambda$-ds-DNA molecules with hom regions longer than $\sim 5 \mathrm{kbp}$, at [salt] and

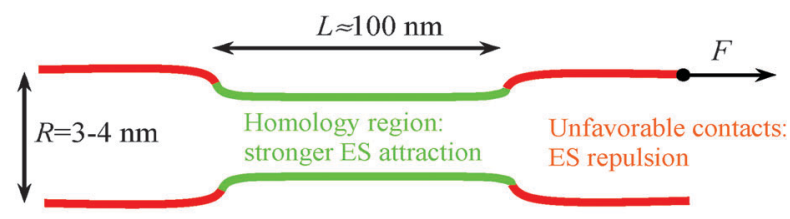

ES recognition energy,

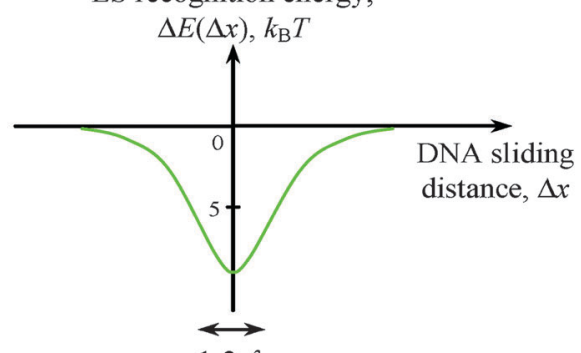

$1-2 \lambda_{\mathrm{c}}$

Fig. 13 Pictorial shape of the ES recognition energy well for the process of sliding of DNAs with a hom domain, as obtained from eqn (1) of ref. 188 for two torsionally rigid DNAs. DNA hom segments are marked in green, while non-hom DNA sections are in red. DNA hom fragments are pinned near the bottom of the well by a stronger ES attraction, relative to the rest of the DNA. Parameters: $\lambda_{c}=100 \AA, R=30 \AA, \theta=0.8, f=0.3,1 / \kappa=7 \AA$ that gives the value $a_{1} \approx 0.015 k_{\mathrm{B}} T / \AA$. The helical coherence length for DNAs in solutions and wet fibers used in this plot, $\sim 10-20 \mathrm{~nm}$, is much shorter than that in DNA crystals, $\lambda_{c}=50-100 \mathrm{~nm},{ }^{84}$ where DNA duplexes are "straightened" by mutual interactions.

[DNA] close to those in vivo. ${ }^{201}$ The paired structures of hom DNAs were sheared by $F \approx 10-20 \mathrm{pN}$ forces and pairing was more profound in the presence of $\mathrm{MgCl}_{2}$, indicative of the ES nature of this effect. Some other properties, such as strong enhancement of pairing efficiency with [simple salt] up to $1 \mathrm{M}$ as well as non-monotonic $T$-dependence favor however rather some non-Coulombic picture for the pairing forces. ${ }^{202}$ Also, in experiments, the precision of hom DNA-DNA register along the paired DNAs is often $\sim 2-5 \mu \mathrm{m}$, being much larger than the width of ES DNA-DNA recognition energy well in the theory, that is $\sim \lambda_{c} \approx 10-50 \mathrm{~nm},{ }^{188}$ see Fig. 13 . The width in experiments is also independent of the length of paired hom DNA segments. Future developments of this highly promising 
technique might provide more information on the axial proximity of paired hom DNAs and thus enable us to infer the effective "action range" of the sequence-specific DNA-DNA forces monitored.

We do believe that DNA-DNA helix-specific ES forces can govern DNA sequence recognition in dense DNA phases, ${ }^{200}$ at high DNA densities and strongly suppressed DNA fluctuations. DNA-DNA hom associations in vivo, are however often maintained at much larger separations and occur between fluctuating DNAs. ${ }^{203,204}$ The pairing remains efficient at DNA-DNA distances much longer than the screening length that inherently limits the action radius of DNA-DNA ES forces. The DNA-DNA hom pairing process should therefore also involve some recognition mechanisms other than the direct DNA-DNA ES forces, ${ }^{205}$ probably including proteinmediated DNA-DNA contacts.

\section{DNA-DNA close-range friction}

Modern bio-nano-tribology applications necessitate a detailed understanding of frictional forces between bio-molecules on the nano-scale. ${ }^{207-209}$ For DNA, recent advances in singlemolecule manipulation techniques have allowed measuring the forces required to pull one DNA over another in a tight superhelical DNA ply, the so-called dual optical trap apparatus. Tight winding of two DNAs in the ply implemented in this set-up can facilitate their interactions. ${ }^{210,211}$

For shearing the DNA ply, in the presence of DNAassociated DNA-bridging H-NS proteins, frictional forces up to $\sim 25 \mathrm{pN}$ were detected ${ }^{212}$ They emerge through disruption of the DNA-protein-DNA bridges formed every several $H$ along the DNA ply. Also, when some small proteins bind to ds-DNAs and sterically impede DNA pulling, the frictional forces of $\sim 2-5 \mathrm{pN}$ are detected. ${ }^{212}$ For "bare" DNAs, one could expect that inherent DNA helicity on the scale of $H=3.4 \mathrm{~nm}$ might itself generate some friction. With the same apparatus, no measurable friction was however detected: ${ }^{213}$ the forces remained $<1 \mathrm{pN}$, independent of the length of the DNA ply, the DNA pulling speed, and the presence of DNA-condensing (spermine ${ }^{4+}$ ) cations in the solution. The diameters of DNA superhelical plies in experiments were estimated as $\sim 5-10 \mathrm{~nm}$.

Using the theory of DNA-DNA ES forces from section 2, we have examined different regimes for DNA-DNA nanofriction depending on the character of the DNA sequence. ${ }^{214}$ For ideally helical non-fluctuating and closely juxtaposed DNAs, DNA-DNA ES friction emerges due to spatial correlations of ES potential along the DNA surfaces, Fig. 14. At relevant salt conditions, this effect is only pronounced in the first $\sim 10 \AA$ from the DNA surface. The ES frictional force in this regime oscillates with a period of $3.4 \mathrm{~nm}$ and its magnitude grows linearly with the DNA length $L$. Namely, the static friction force is $F_{f r}=2 \pi a_{1} L / H$. For a slow DNA pulling speed, this gives rise to a stick-slip motion on the nanoscale. ${ }^{214}$

DNA-DNA friction however remains rather low. Even for very tight DNA plies, with diameters of $2 R=40 \AA$ and for DNA parameters favoring DNA-DNA ES attraction

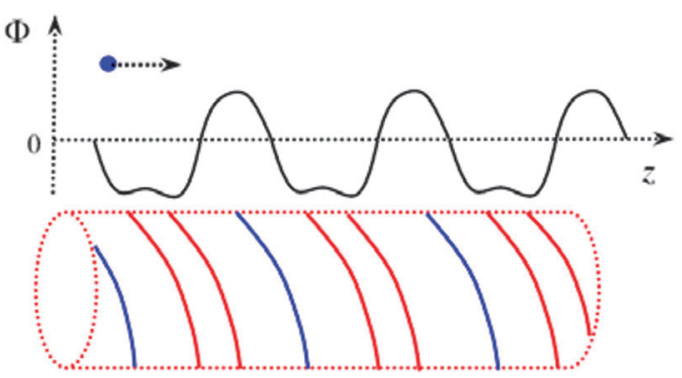

Fig. 14 Schematics of ES potential barriers $\Phi(z)$ near the DNA surface. The negative DNA phosphate strands are shown in red, the counterions adsorbed in the DNA major groove are depicted as blue helices.

(larger $a_{1}$ values), the upper estimate for possible friction for a ply with $N=L / H=10$ DNA turns is only $\approx 4 \mathrm{pN}$.

Several effects are likely to reduce this upper limit even further. It is the case in the model of pulling non-ideally helical DNAs that are realized for non-related bp-sequence. For such molecules, the "corrugations" in the DNA helical structure progressively accumulate with the DNA length ${ }^{80}$ and the ES potential variations along the DNA-DNA contact become de-correlated, as discussed in section 6 . This, in turn, strongly impedes DNA-DNA ES friction that attains in this limit an exponential decay with the pulling distance of one DNA with respect to another in the ply. As the DNA-DNA ES interactions decay exponentially with separation $R$, it is not surprising that for DNA plies that are typically much thicker than $2 R \approx 40 \AA$, being formed in solution by thermally fluctuating DNAs with quasi-random sequences, no measurable friction has been detected in DNA-pulling experiments. ${ }^{213}$

The situation might however change, when tighter DNA plies are realized (for instance, by larger static stretching forces applied to DNA ends) and for special DNA sequences with some degree of bp-homology. One example is the DNA molecules designed to contain repetitive bp hom blocks with a length of $\sim 50-300 \mathrm{bp}$. Then, one could expect some homologymediated DNA pinning events upon DNA pulling at the positions when these hom blocks on two DNAs overlap the most. Theory predicts $^{188}$ that these pinned states have the half-width of $\sim \lambda_{c}$, see Fig. 13. It makes a detection of such a pinning more amenable to the current experimental technique ${ }^{213}$ with a resolution of several DNA helical repeats $H$. On the contrary, the resolution of at least $H / 2$ is necessary to probe the ES DNA-DNA friction predicted above, that stems from DNA helicity on the length-scale of $3.4 \mathrm{~nm}$.

One potential effect of the predicted DNA-DNA friction is on DNA ejection from ds-DNA phages, when astonishingly densely packed DNA strands have to slide passing each other upon reorganization of DNA layers inside the capsid, see section 12 .

\section{DNA melting and hybridization in dense lattices}

Upon heating up to $\approx 50-100{ }^{\circ} \mathrm{C}$, depending on the GC-content and bp sequence, DNA molecules melt cooperatively in solution and their strands separate. Being thoroughly studied at low [DNA], see classical ${ }^{215,216}$ and also recent studies, ${ }^{217-221}$ 
the phenomenon of DNA melting in dense assemblies, when intermolecular forces become comparable to the internal DNA binding energies, remains not completely understood. Some ES effects on DNA melting and hybridization phenomena in dense DNA lattices are discussed below.

\section{A DNA melting}

The effects of DNA sequence on DNA melting in dense hexagonal assemblies are discussed here, within a simple thermodynamical model developed in ref. 222. In particular, it is straightforward to show that under conditions favoring duplexduplex attraction, ideally helical hom DNAs melt at higher $T$ due to stabilization of ds-regions ${ }^{223}$ mediated by DNA-DNA ES attraction. For hom DNA fragments, our model predicts a rise of the melting temperature $T_{m}$ (typically by $3-10^{\circ}$ at $R=23-28 \AA$ between DNAs in the assembly) and more cooperative DNA melting transition. The shift of $T_{m}$ scales with the strength of DNA-DNA ES attraction, namely $\Delta T_{m} \propto 3\left|a_{0}-a_{1}\right|$. It can thus be controlled in feasible future melting experiments in DNA dense phases via addition of attraction-mediating cations, e.g. with enhanced binding into the DNA major groove.

Also, the model predicts a change in the character of melting transition, from the second- to the first-order at a critical strength of DNA-DNA attraction, ${ }^{222}$ see Fig. 15. Then, at the melting point the fraction of helical DNA regions exhibits a jump-like change. At the isotropic-to-cholesteric DNA phase transition, at a moderate [DNA] of $\approx 100-150 \mathrm{mg} \mathrm{ml}^{-1}$, a clear indication of $T_{m}$ jumps by several degrees was observed and quantified already long ago. ${ }^{224}$

Recently, for dense aggregates of 10-50 nm GNPs linked by short DNA fragments ${ }^{225}$ an extremely sharp DNA melting transition has also been monitored, with a width of transition of $1-2^{\circ}$ as compared to $\sim 10-20^{\circ}$ for DNA melting in solution. This enhanced DNA melting cooperativity was attributed to short-range DNA-DNA interactions that trigger an

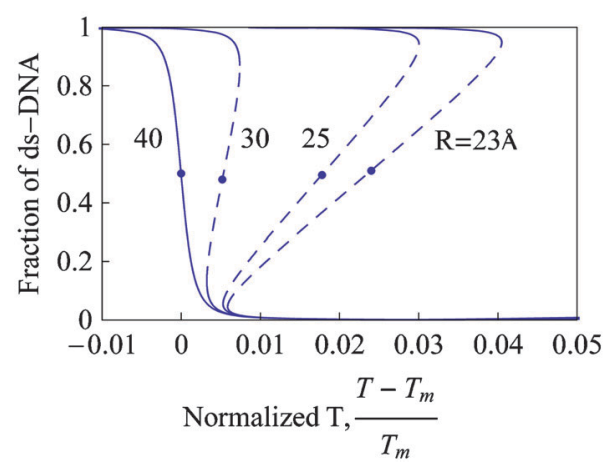

Fig. 15 Melting profiles predicted for long hom DNAs in the hexagonal assembly at varying DNA densities. The dots on the curves indicate the melting temperatures for a corresponding first-order transition. At this point, an abrupt charge in DNA helicity occurs between stable branches of DNA melting curve, due to a Z-shaped DNA melting isotherm realized at large enough DNA-DNA attraction strengths. The parameters for DNA-DNA ES forces are the same as in Fig. 13. The figure is reprinted from ref. 222, subject to ACS-2005 Copyright. accumulation of ions from electrolyte in the overlapping ES double layers around the molecules, ${ }^{226}$, in a Donnan-like fashion. For dense DNA bundles connecting DNAfunctionalized GNPs, ${ }^{227}$ with DNA-DNA distances of $R=$ 25-40 $\AA$, a higher local [salt] near the DNAs are realized, which in turn tends to stabilize the DNA ds-state and rise the melting temperature. The effective increase of $T_{m}$ at these DNA densities was calculated to be $5-20^{\circ},{ }^{226}$ based on a linear increase of $T_{m}$ on $\log [[\mathrm{salt}]] .{ }^{225}$ Once the melting of DNA bundle connecting two GNPs starts, it progressively releases the excess counterions thus destabilizing the remaining ds-DNAs. This might cause sharp melting of the entire GNPs-DNAs assembly, as indeed detected in experiments. ${ }^{225}$ Recently, sharper melting and higher $T_{m}$ values have also been measured for a dense DNA hybrid of just a pair (!) of short DNA duplexes. ${ }^{228}$

Our DNA melting model for dense aggregates of identical DNA helices does account for the Donnan equilibrium in the DNA lattice, that would generate a corresponding $\left[\mathrm{Na}^{+}\right]$induced rise of DNA melting temperature. ${ }^{229}$ The additional $T_{m}$ shifts illustrated in Fig. 15 are however solely due to DNA-DNA sequence-specific ES attraction that appears also to be capable of inducing abrupt changes in the average DNA helicity.

For bp random ds-DNA sequences externally pressurized to form dense DNA assemblies, one expects ${ }^{222}$ on the contrary destabilization of ds-DNA by DNA-DNA ES length-dependent interactions. ${ }^{80}$ The physical mechanism here is more formidable. Namely, the melted ss domains/bubbles are going to be created on ds-DNAs to optimize unfavorable ES repulsions predicted to occur for bp-random DNA stretches longer than $1-2 \lambda_{c},{ }^{82}$ see the dot-dashed curve in Fig. 12a. The melting transition becomes less cooperative in this case. An experimental support of some of these theoretical findings might come from recent $T_{m}$-measurements in strongly confined wet DNA films. ${ }^{230}$

\section{B DNA hybridization and detection}

One way to detect DNA strand separation and hybridization is to monitor the signal changes of a charge-responsive biosensor ${ }^{231}$ functionalized with arrays of ss-DNA molecules. Recently, an ES theory of signal generation on the surface of a field-effect biosensor upon DNA hybridization on relatively dense lattices of end-tethered probe ss-DNAs has been developed in our group. ${ }^{232}$ The sensor surface was first functionalized in experiments with a dense layer of GNPs of 4-6 nm in diameter. Each GNP was then decorated by 2-5 probe ss-DNA fragments of 20-30 bp in length. The procedure allowed us to enhance the rates of DNA hybridization considerably, in agreement with recent predictions. ${ }^{233}$

The range of model parameters was determined when the ES potential variations predicted on the sensor surface upon DNA hybridization (by linear and non-linear PB models) agree with experimentally measured $90-120 \mathrm{mV}$ of voltage change. The key quantities are the density of ss-DNA lattice on the sensor surface and effective counterion concentration in solution between ss- and ds-DNAs. To improve the reproducibility of sensor signals and reduce the effects of disturbing factors, the detection of DNA hybridization in a differential mode was 
performed. $^{232}$ It was carried out at high [salt] during the DNA hybridization process and at low [salt] upon the readout of the sensor output signals.

For situations, when the probe ss-DNA lattice is immobilized directly on the sensor surface as dense DNA micro-arrays, ${ }^{234}$ the ES effects upon hybridization of complementary target ss-DNAs from solution have also been elucidated. ${ }^{235}$ Here, the ES effects primarily originate from redistribution of mobile ions via the Donnan equilibrium. Both techniques can be employed for a fast and cheap label-free detection of relatively short DNA fragments from unknown DNA mixtures, suggesting new ways of controlling hybridization kinetics and efficiency, as required for various biological and biomedical applications.

Note that the experimental apparatus developed ${ }^{232,235}$ is only amenable for implementation for relatively short DNA fragments, $L<30-50 \mathrm{bp}$. DNA micro-arrays of short oligonucleotides allow more precise control over a single-bp DNA sequence mismatch and ensure fast DNA hybridization kinetics. The hybridization efficiency for longer DNA sequences is reduced considerably, together with the accuracy of output signals. Positive voltages applied on the sensor surface might speed up the kinetics of ss-DNAs association and improve the regularity of ds-DNA lattices generated after DNA hybridization. ${ }^{236}$ Relatively high DNA lattice densities are required for controllable read-out of signal differences upon DNA hybridization, typically $\sim 10^{15}-10^{17} \mathrm{ss}-\mathrm{DNAs} / \mathrm{m}^{2}$. Efficient hybridization of longer sequences becomes problematic at these ss-DNA densities, because of steric hindrance of probe and target DNA fragments and the ES constraints imposed.

\section{DNA-protein ES recognition: models and reality}

\section{A Protein-DNA recognition}

Despite enormous experimental and theoretical efforts in the last decades, the laws governing recognition of DNA-binding proteins and their cognate sites on ds-DNA still remain obscure. The shape complementarity of protein $\alpha$-helices fitted into the DNA major groove ${ }^{237}$ and protein-DNA charge matching ${ }^{238}$ often drive the formation of complexes. Several types of interactions might contribute to protein-DNA binding, with ES, HB formation, and hydrophobic contacts being often the dominant ones The formation of non-specific and specific complexes of a protein with DNA can be directed by interactions of different types. Protein structures and their DNA recognition domains are extremely diverse which makes it extremely complicated to establish universal principles of the DNA-protein recognition.

A number of small DNA-binding proteins (transcription factors) can locate their targets on DNA with rates that are much faster than allowed by the thermal diffusion in 3D solution, e.g., up to $10^{2-3}$ times faster for the lac repressor. ${ }^{239,240}$ Being capable of such speedy scanning of DNA, these proteins often recognize DNA sequences with high precision, tolerating little bp-mismatch. We address the reader to recent perspectives on facilitated protein diffusion on DNA, ${ }^{241-246}$ without discussing many facets of this interesting dynamical problem here.

The ES forces are known to dominate the non-specific binding for a number of DNA-protein complexes, e.g. weakly bound lac repressor. ${ }^{247-249}$ The DNA-binding domains of many small DNA-binding proteins contain positively charged patches that ensure the recognition of features in DNA ES surface potential and DNA-protein ES attraction, ${ }^{250}$ even for net negatively charged proteins such as the lac repressor. For relatively large protein assemblies, the situation is quite similar. For RNA Polymerase II, for instance, a strongly positively charged cleft/saddle has been identified in the crystal structure along a path taken by DNA-RNA hybrid upon transcription. ${ }^{31}$ Also for the ribosome, the basic protein residues are located in the structure protrusions expected to be involved in the binding of tRNA/mRNA during translation. ${ }^{30}$

Indeed, $\mathrm{Lys}^{+}$and $\mathrm{Arg}^{+}$residues in DNA-protein complexes are often located within only several $\AA$ from negative DNA phosphates, see Fig. 16. ES DNA-protein interactions are however believed to bear little specificity to DNA sequence, ${ }^{251}$ just providing a protein-DNA proximity and allowing more sequence-specific and orientation-dependent $\mathrm{HB}$ contacts to recognize the patterns of $\mathrm{HB}$ donors and acceptors inside DNA bases. $^{252}$ It is however hard to guess a priori to what extent this conjecture is valid for a particular DNA-protein complex.

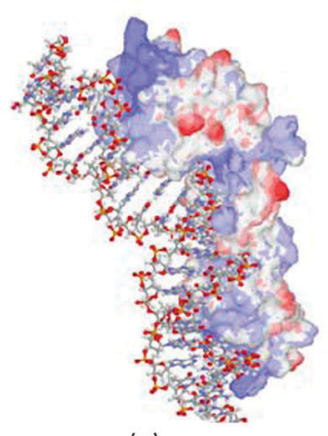

(a)

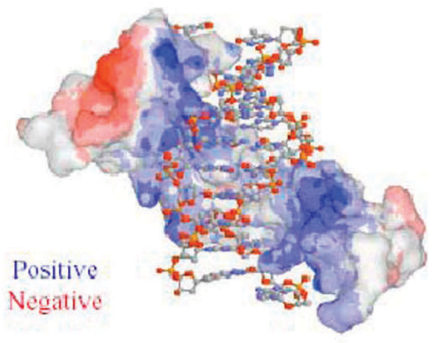

(b)

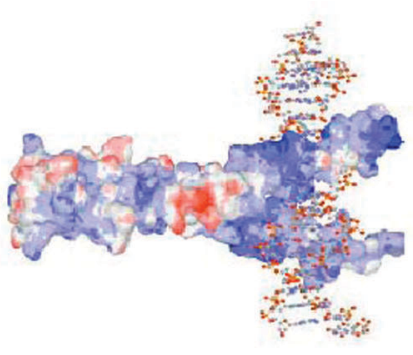

(c)

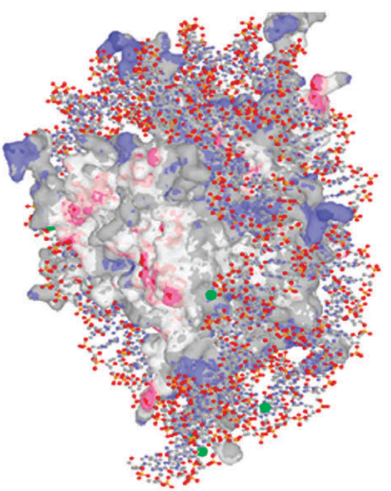

(d)

Fig. 16 The distribution of ES potential on DNA-protein complexes of specifically bound lac repressor 111m.pdb (a), zinc finger ZIF268 1aay.pdb (b), leucine zipper GCN4 1ysa.pdb (c), and 146 bp NCP 1aoi.pdb (d). The structures are visualized by MDL Chime and Protein Explorer, using the PDB files of complexes. Images are not to scale. 


\section{B Analytical results}

To have some rough estimates at hand, a simple 1D physical model of DNA-protein ES recognition based on the complementarity of their charge patterns has been constructed. ${ }^{253}$ DNA and protein charge lattices were set to fully commensurate for the cognate site being de-correlated for all other sequences on the DNA, see Fig. 17. In the model, some random charge displacements along the DNA $\Delta_{n}$ and protein $\delta_{m}$ axes mimic the sequence specificity of charge positioning on DNA and on the protein. Our "model protein" is thus attracted stronger to this "commensurate" DNA segment. ES recognition energy well near this target site has been derived based on the linear PB theory. ${ }^{253}$ For typical parameters (a dozen of positive charges on a protein that are about $R=1 \mathrm{~nm}$ away from the DNA) the recognition well amounts to $\approx 3-10 k_{\mathrm{B}} T$ in depth and a couple of $\mathrm{nm}$ in width, see Fig. 18. Therefore, this short-range ES well cannot serve as a "funnel" that would direct the diffusing proteins from far away on the DNA to this charge-hom binding site. This well thus does not contribute strongly to a facilitation of protein diffusion and other dynamical mechanisms have to be responsible for it.

In this linear ES model, the depth of the ES well scales linearly with the number of charges in the homology domain. It is also proportional to the magnitude of charge deviations from their quasi-periodic positions on the lattice, described by parameter $\Omega^{2}=\left\langle\Delta_{n}{ }^{2}\right\rangle+\left\langle\delta_{m}{ }^{2}\right\rangle$. At [salt] $=0$, the well depth decreases with the protein-DNA separation $R$ as $\propto 1 / R^{3}$, while in the presence of screening the decay is exponential, $\propto e^{-R / \lambda_{D}}$. For weak charge fluctuations and in the absence of salt the model returns an elegant expression for the ES recognition energy well ${ }^{253}$

$$
\Delta E(\Delta z)=-\frac{k_{\mathrm{B}} T l_{B} M \Omega^{2} \varepsilon}{2 \varepsilon_{c}} \frac{R^{2}-2 \Delta z^{2}}{\left(R^{2}+\Delta z^{2}\right)^{5 / 2}} .
$$

Here $M$ is the number of charges in the hom domains and $\Delta z$ is the mutual protein-DNA sliding distance with respect to complete homology overlap at $\Delta z=0$.

Similar to exact calculations, this expression also features barriers on both sides of the recognition well. In this 1D model, the well for the complete match of the DNA and protein hom lattices is accompanied by energetic barriers. These disappear in a model with charge displacements perpendicular to the DNA-protein plane. ${ }^{254}$

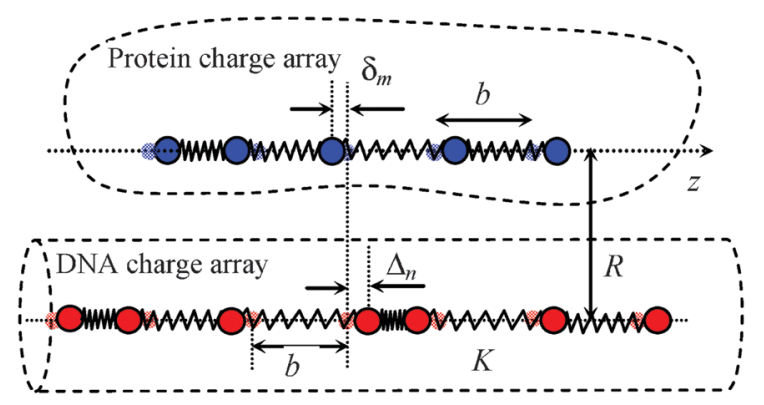

Fig. 17 Schematic 1D-model of "protein-DNA" ES recognition. The charge positions on the DNA and protein vary in a random fashion prescribed by $\delta_{m}$ and $\Delta_{n}$ about the quasi-periodic positions on the 1D lattice $z_{n}=n b$.

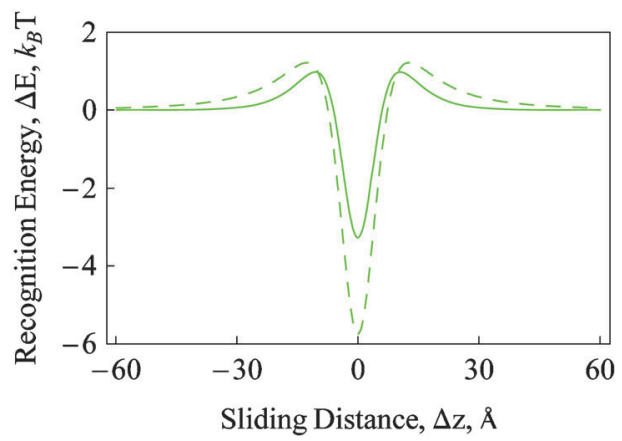

Fig. 18 ES recognition energy well upon sliding of "protein" over "DNA" lattice with the homology section at $\Delta z=0$. Parameters: $M=11$ charges in hom region, DNA-protein separation is $R=10 \AA$, $\Omega^{2}=2 \AA^{2}$. The charges are assumed to interact through a weaklypolarizable low-dielectric medium between DNA and the protein with a dielectric constant $\varepsilon_{c}=2$. The dashed curve is the zero-salt limit, the solid one is for $1 / \kappa=7 \AA$ when the recognition energy is reduced.

The protein-DNA ES recognition well then resembles the DNA-DNA barrier-free ES hom recognition well sketched in Fig. 13. Random twist "fluctuations" of DNA charges occur also perpendicular to the DNA-DNA plane. Generalization of this 1D model for protein and DNA charge displacements for $2 \mathrm{D} / 3 \mathrm{D}$ situation is more realistic and computationally feasible. Some specific and not fully random charge displacement laws can mimic interactions between particular charge patterns. The recognition energies in this case become however hard to handle analytically. Note that the calculation of this DNA-protein recognition well is methodologically similar to that for the DNA-DNA ES recognition funnel discussed above. $^{188}$

A short-range ES well derived is capable of slowing down the protein diffusion, provoking protein trapping for $\sim \mu \mathrm{s}-\mathrm{ms}$ near this "hom" site on the DNA. ${ }^{253}$ This time is enough to trigger some conformational changes in the protein and DNA structures that might induce stronger (chemical or HB) protein binding to this particular DNA fragment. Our hypothesis is a two-step mechanism of recognition for some DNA-protein complexes. First, a DNA-binding protein scans the DNA ES surface looking for a charge-complementary site. In this "searching" mode, the protein structure is flexible and adaptable to the lattice of interaction sites on the DNA. When the commensurate DNA fragment is found, the interaction-induced folding solidifies the protein structure switching it into a "binding" mode that enables stronger/specific contacts with DNA. ${ }^{3}$ This locus on DNA might serve as a promoter sequence for initial pause/binding of processive DNA-binding proteins such as RNA Polymerase and also provide specific targets for binding of transcription factors such as the lac repressor. Cumulative $\mathrm{ES}$ and $\mathrm{HB}$ contacts form rigid specifically bound DNA-protein complexes.

A similar two-step recognition mechanism was recently observed for some zinc-finger proteins. ${ }^{255}$ Using the crystal structures and molecular dynamics simulations, a pre-organized assembly of protein side-chains was detected that forms an ES "hot spot" for recognition of the interior of DNA grooves. Only DNA-protein complexes with this ES pocket were found to form tightly bound structures, first via non-specific interactions 
of amino acids with DNA phosphates that are later amplified by HB formation with DNA bases. ${ }^{255}$

Such two-step docking is also present for some protein-protein complexes. The assembly pathway into the native structure is directed by "anchoring" of a shape-complementary relatively rigid "key" domain of one protein into a "lock" domain in the surface of another protein. ${ }^{256}$ This process is accompanied by a large burial of solvent accessible surface area and water release. This amplifies further docking of protein surfaces into a tight complex.

\section{Bioinformatic analysis of PDB structures of DNA-protein complexes}

To validate this analytical protein-DNA recognition model ${ }^{253}$ for different classes of DNA-binding proteins, an analysis of biochemical structural details available via the PDB for proteinDNA crystal structures has been performed. The distribution of $\mathrm{NH}_{2}{ }^{+}$groups on $\mathrm{Arg}^{+}$and $\mathrm{Lys}^{+}$residues in the DNA-binding domains of DNA-protein complexes has been analyzed. ${ }^{257}$ In particular, large structural complexes were studied, such as the NCPs of eukaryotes ${ }^{5}$ and the architectural proteins of prokaryotes, ${ }^{258}$ both involving an extensive DNA wrapping around the protein cores. The DNA binding in these complexes is known to have a large ES contribution.

Using a home-written computer code for extracting the coordinates of the DNA phosphate groups $\mathrm{PO}_{4}{ }^{-}$and protein $\mathrm{N}^{+}$and $\mathrm{O}^{-}$charges from the PDB files, we could analyse the distances from $\mathrm{N}^{+}$atoms on $\mathrm{Arg}^{+}$and $\mathrm{Lys}^{+}$that are within $\approx 7 \AA$ from the closest $\left(s_{1}\right)$ and next closest $\left(s_{2}\right)$ DNA phosphates on the same strand, see Fig. 19. The statistics of the ES contacts and salt bridges in DNA-protein complexes can thus be restored. Smaller cut-off distances of 3-5 $\mathrm{A}$ can also be used in the analysis, to minimize the contribution of DNA charges from the neighboring strand across the DNA narrow groove. Note that fluctuation-induced uncertainties in the positions of protein charges in crystals of DNA-protein complexes are often $\sim 1-2 \AA$, still much smaller that the relevant periodicity in the problem, the $\mathrm{P}^{-}-\mathrm{P}^{-}$distance $s_{\mathrm{ph}} \approx 7 \AA$ along the B-DNA helical strand.

Histone positive charges in the NCPs are mainly localized in the outer "ring", close to wrapped DNA, see Fig. 16d, while negative $\mathrm{Asp}^{-}$and $\mathrm{Glu}^{-}$residues are rather inside the NCP core. Our analysis demonstrates that $\mathrm{N}^{+}$atoms on $\mathrm{Arg}^{+}$and $\mathrm{Lys}^{+}$track the positions of individual DNA phosphates, as visualized in the histogram of $s_{1}-s_{2}$ for all $\mathrm{N}^{+}$charges in the DNA vicinity, Fig. 20. The bimodal distributions were detected both for individual NCPs (good statistics can be achieved for a single particle) and for the entire family of 146 bp long complexes. ${ }^{257}$ This indicates that $\mathrm{N}^{+}$on Lys and Arg are encountered more often close to one of DNA $\mathrm{P}^{-}$ charges than between the two, maximizing thereby the ES attraction of this imprinted pattern of charges to the DNA. As the DNA structure and positions of the DNA phosphates are strongly correlated to the bp sequence, ${ }^{79}$ the detected "charge tracking" for particular DNA sequences wrapped in the NCPs yields a conclusion about sequence-specificity of DNA-protein ES interactions. This supports our model hypothesis about commensurate charge lattices on the DNA target

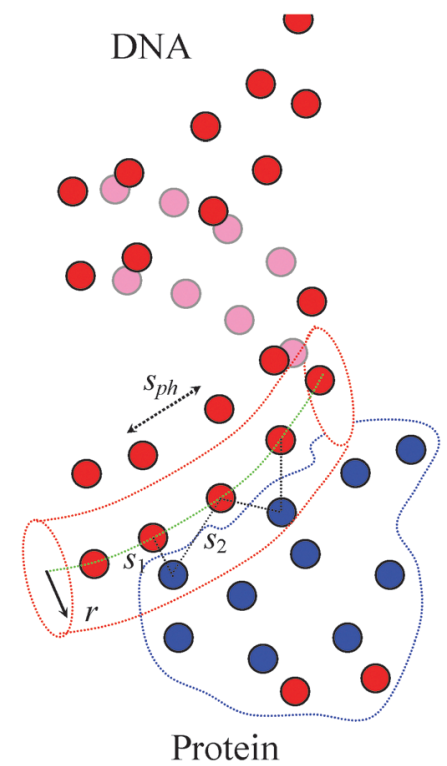

Fig. 19 Definition of $s_{1,2}$ distances for protein positive charges (in blue) which are closer than $r \sim l_{B} \approx 7 \AA$ to negative DNA phosphates (red helix).

sequence and on the protein. For NCPs, this fact can contribute to NCP positioning on genomic DNAs, ${ }^{259}$ interfering with the known mechanism of sequence-specific DNA bendability believed to govern this process. ${ }^{260,261}$

A specificity of the ES binding of histones' Arg and Lys in DNA grooves has recently been also discovered by other groups. ${ }^{262}$ The ES-directed localization of $\mathrm{N}^{+}$from $\mathrm{Arg}$ in the minor grooves in AT-rich DNA regions was confirmed by a detailed analysis of a number of DNA-protein complexes also in ref. 263. It was emphasized that for DNA sequences wrapped in NCPs the AT-tracts have particularly narrow minor grooves due to the spatial proximity of negative DNA phosphate strands, forming thus "attractive" sites for $\mathrm{Arg}^{+} /$ $\mathrm{Lys}^{+}$binding. Arg was claimed to be preferred over Lys in the minor grooves because of a lower self-energy cost to remove a larger guanidinium group of $\mathrm{Arg}^{+}$from its hydrated state in solution and bring it in contact to the DNA, than to do the same for a smaller ammonium group of the $\mathrm{Lys}^{+}$residue. ${ }^{263}$ The reason is the Born ES self-energy that scales inversely

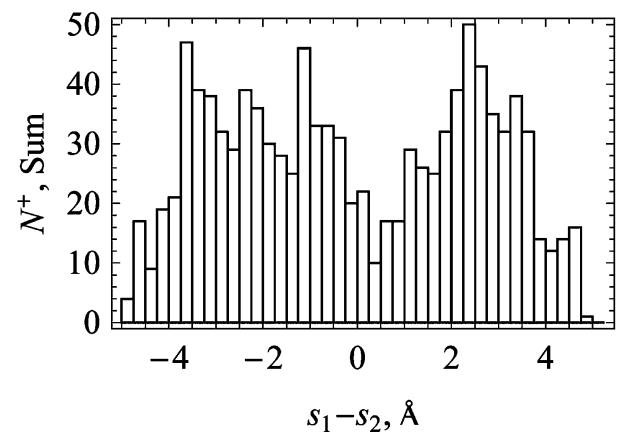

Fig. 20 Bimodal distribution of $s_{1}-s_{2}$ distances for in total 14 NCPs that indicates ES recognition of individual DNA phosphates by the closest $\mathrm{N}^{+}$atoms on Arg and Lys residues of histone proteins. 
proportional to the "ion" radius ${ }^{264-268}$ (see also recent numerical studies on the hydration of small organic molecules ${ }^{269,270}$ and polypeptides $^{271-273}$ ).

Being valid for large structural complexes, the ES complementarity recognition model however fails for small DNAprotein complexes, with simple standard motifs of DNA recognition (e.g., helix-turn-helix, zinc finger, and leucine zipper). For a large set of small proteins from these families, we could not detect any statistical preference in the distribution of $\mathrm{Lys}^{+}$and $\mathrm{Arg}^{+}$ close to DNA phosphates in protein-DNA complexes. To make a more definite conclusion, some redundant structures are to be excluded from the analysis and a grouping into smaller, more specific protein sub-families is to be performed.

As a tentative explanation one can suggest however that for large complexes, with $\sim 30-100$ ES DNA-protein contacts, the ES energy gain being ES-commensurate can reach $\sim 10-30 k_{\mathrm{B}} T$ and proteins appear to use it for sequence-specific binding to DNA. For small protein-DNA complexes, with only 3-10 ES contacts and much weaker ES binding, other interactions (such as HBs) are likely to direct the recognition of specific DNA sequences.

\section{Approximations and perspectives}

It is interesting to mention that ES DNA-protein commensurability for the NCPs and their prokaryotic analogs resembles a zipper-like positioning of positive and negative amino acids along the interfaces of many protein-protein complexes. ${ }^{23,274}$ Long-range ES attractive forces between commensurate charge patches on the protein surfaces were shown indeed to enhance the protein-protein association in solutions, ${ }^{275-277}$ being capable to achieve rates of $10^{10-11} / \mathrm{M} / \mathrm{s}$, often beyond the diffusion-controlled limit. These rates are close to the optimal association rates measured for a specific binding of the lac repressor to its operator site on long (!) DNAs at $0.1 \mathrm{M}$ of simple salt. ${ }^{239}$ These rates are achieved however without a presence of a 1D-track for protein diffusion like in facilitated association of proteins with a target on DNA. For proteinprotein association, despite the hydrophobic residues often dominating the overall binding affinity, ${ }^{278}$ these non-charged amino acids might be too abundant to provide proper binding specificity. ${ }^{238}$ The latter might in turn stem from charge patchiness and propensity of $\mathrm{HB}$ formation between the residues along the contact surface of the bound proteins. ${ }^{279,280}$

Several issues offer a perspective for future studies. First, for evaluation of ES binding energies for DNA-protein complexes, on top of the statistical analysis of charge patterns described above, one needs to guess ${ }^{281,282}$ a rather small dielectric constant $\varepsilon_{c}$ in the space between DNA and protein. Because of dielectric saturation effects in strongly confined waters hydrated on the charged objects, its value can vary widely, $\varepsilon_{c} \sim 2-30,{ }^{13,16}$ so does the ES interaction energy. ${ }^{283}$ Another important ES issue is the actual charged state of ionizable protein groups in a particular environment of DNA-protein complexes, with the $\mathrm{p} K_{\mathrm{a}}$ value being affected by the local ES potential, [salt], local dielectric permittivity, etc. ${ }^{13,284,285}$

Non-ES van der Waals and HB contacts, as well as the entropic terms associated with water release and counterion evaporation upon DNA-protein binding, are to be quantified in the future models as well. To make a definite conclusion about the mechanism of binding specificity ${ }^{286}$ for a given DNAprotein complex, the ES preference of Arg/Lys positioning with respect to DNA phosphates has to be accompanied by the analysis of $\mathrm{HB}$ formation propensity between protein residues and the sites inside DNA bases. ${ }^{252}$ Also, one has to keep in mind that the protein (and DNA) structures in crystals exposed to special crystallization buffers ${ }^{287}$ might measurably differ from structures stable in physiologically relevant solutions.

There exists an opinion in the literature that ES contacts of charged residues in protein-DNA complexes ${ }^{4}$ and along protein-protein interfaces ${ }^{13}$ might actually destabilize their binding. Namely, a release of structured and ES-favored $\mathrm{H}_{2} \mathrm{O}$ shells around the constituents often accompanies the complex formation. ${ }^{4,288}$ And, it is possible that protein and DNA charged groups complexed together via ES attraction do not fully compensate for energetic loses upon their "ES desolvation". The latter depends crucially on the $\varepsilon$-value assigned to a protein and its surface. For DNA-protein complexes, the entropic effects of released condensed cations from DNA, with the number defined by the slope of log[binding constant] on $\log [\mathrm{salt}]$, are often presented as the main driving force for complexation. Here, the situation is rather similar to counterion release from DNA-(CL membrane) complexes considered in section 4 . In both cases, we however tend to think that the direct ES attraction between the oppositely charged system components governs the complex formation, rather that a concomitant entropic free energy gain due to release of condensed counterions.

\section{DNA wrapping in NCPs}

NCP is a basic unit of DNA compactification in eukaryotic genomes. The octamer of histone proteins has an overall charge of $Q \approx+150-230 e_{0}$ (depending on many factors) and $\approx 146 \mathrm{bp}$ DNA fragment with a bare charge of $\approx-300 e_{0}$ wraps around it in a left-handed super-helical manner. ${ }^{5}$ The ES forces largely dictate the complexation of these highly charged components. NCPs are known to be stable only in a limited range of [simple salt] close to $\sim 0.1 \mathrm{M} .{ }^{289}$ At smaller [salt] the DNA becomes stiffer due to ES contribution to its bending persistence length, ${ }^{290,291}$ see also ref. 292-296. At high [salt], on the other hand, the ES DNA-(histone core) affinity is attenuated by screening. Both tendencies disfavor DNA wrapping into stable NCP particles. The positive charge of the histone core (without highly basic histone tails and $\mathrm{H} 1$ linker histones) is thus $\sim 30-50 \%$ smaller than the DNA negative charge.

The idea of NCP overcharging based on asymmetric DNA charge neutralization, which generates a spontaneous DNA curvature towards the core, was suggested long ago, ${ }^{297}$ see Fig. 21. The possibility of spontaneous DNA bending has been confirmed experimentally for DNA fragments designed so that the phosphate groups are neutralized on one side of the DNA. $^{298}$ Recently, the subject of PE-sphere wrapping and induced overcharging ${ }^{299}$ has been investigated in a number of computer simulation ${ }^{300-304}$ and theoretical ${ }^{305}$ studies. 


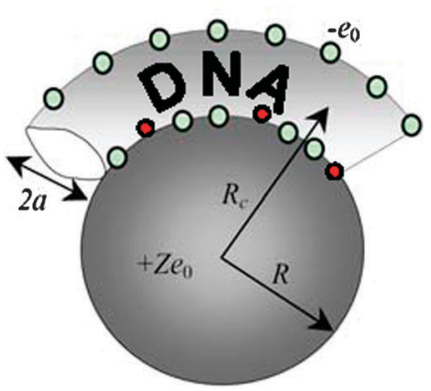

Fig. 21 Model for NCP overcharging by a wrapped PE chain with a finite diameter.

Experimentally, the DNA complexation with cationic poly-lysine coated nano-spheres, intended to mimic the DNAhistone assembly, has revealed however strongly undercharged complexed for the particles of NCP-relevant size. ${ }^{306}$ Several mean-field PB theories were implemented to study the complexation of semiflexible PEs with oppositely charged spheres, as a model for NCP formation. ${ }^{307,308}$ Some analytical studies predicted a gigantic, up to 10-30 times, sphere overcharging by a thin PE chain wrapped around it, at an optimal [salt] of $\sim 0.1 \mathrm{M}^{309}$ More accurate ES PB modelling of complexes of thin PEs with oppositely charged spherical ${ }^{310}$ and cylindrical ${ }^{311}$ macro-ions however gave rise to a more physical conclusion, namely that undercharged or close to charge-neutral complexes are energetically favored.

For a PE of finite thickness, a quantitative model of NCP overcharging has been constructed by asymmetrically neutralized semiflexible PE chain. ${ }^{312}$ It allowed to analyse the point of PE wrapping-unwrapping transition in terms of $\mathrm{PE}$ persistence length and [salt]. Realistic DNA elastic parameters, DNA and histone charges, and some mobility of protein charges were taken into account. The model demonstrated that ES repulsion of non-compensated charges on the outer DNA/PE surface provokes its spontaneous wrapping, with the final complex overcharging degrees relevant to NCPs.

\section{NCP-NCP ES interactions and $30 \mathrm{~nm}$ chromatin fibers}

\section{A DNA compaction into chromatin}

NCP-mediated DNA compaction in eukaryotic chromatin provides the physical basis for enormous $10^{4-6}$ compactification "power" often required to pack meter-long genomes in $\sim \mu \mathrm{m}$-sized cell nucleus. ${ }^{24}$ This process takes place on several hierarchical levels, with the initial stage being the so-called 30-nm chromatin fiber, an organized and structured array of NCPs connected by a single DNA strand. The structure and regularity of $30 \mathrm{~nm}$ fiber is highly sensitive to many biologically relevant factors: ionic environment (including the balance of mono-, di-, and multivalent cations ${ }^{25}$ ), DNA linker length, ${ }^{313}$ the concentration of linker histones because of pronounced effects of $\mathrm{NCP} / \mathrm{H} 1$ charge stoichiometry, ${ }^{314,315}$ the charge state of histone tails, ${ }^{316}$ etc. It is still debated what conditions favor solenoidal $^{317}$ vs. zig-zag $^{318,319}$ arrangements of NCPs in chromatin fibers for an array of identical NCPs. Inherent randomness in structure of histone cores and poly-dispersity of DNA linker lengths render the native chromatin fiber structures even more irregular. Some groups even doubt the existence of regular chromatin fibers in vivo. ${ }^{320}$

On higher levels of eukaryotic chromatin compaction, ${ }^{321-324}$ the fiber structure becomes even less certain. ${ }^{325}$ Fiber organization in condensed metaphase chromosomes for instance involves a variety of different proteins. The list includes the structure maintenance ${ }^{326}$ proteins, condensin (with often highly basic DNA binding interface ${ }^{327}$ ) and cohesin, as well as chromosome scaffolding proteins. Different models of DNA compaction on this scale exist, including various looping scenarios. In the interphase, when eukaryotic chromosomes are largely de-compacted, one can distinguish hetero- and eu-chromatin that differ in the density of compaction of genetic material and levels of DNA transcription. During chromosome de-condensation, a number of proteins are likely to be involved in the process too. In addition, upon transition from interphase to metaphase, the concentrations of $\mathrm{Ca}^{2+}$ and $\mathrm{Mg}^{2+}$ cations in the cytoplasm are known to rise several times ${ }^{25}$ pointing out a possible ES pathway of chromosomal compaction via counterionmediated DNA-DNA and NCP-NCP interactions.

In vitro, recent fiber reconstitution experiments provided a great deal of essential information about inherent stability, NCP linear density, and, rather important, on diameters of chromatin fibers for various lengths of DNA linker. ${ }^{328}$ The H1-containing fibers visualized by the cryo-EM have indeed supported the solenoidal model, with the fiber diameter varying with DNA linker length (being $34 \mathrm{~nm}$ for 30-60 bp and $44 \mathrm{~nm}$ for 70-90 bp long DNA linkers). High degrees of fiber axial compaction are achieved via inter-digitated assembly of neighboring NCPs on the fiber periphery, maintained via favorable $\mathrm{NCP}-\mathrm{NCP}$ interactions. The energetics of DNA-histone, ${ }^{329} \mathrm{H} 1-\mathrm{NCP},{ }^{330}$ and $\mathrm{NCP}-\mathrm{NCP}^{331}$ contacts is therefore vital for physical understanding of fiber structure and stability.

\section{B Dense phases of NCPs}

Similar to unwrapping of DNA toroids, that enabled to rationalize their stability, ${ }^{124}$ recent measurements of forceinduced stretching of chromatin fibers revealed the NCP-NCP cohesive energy of $\approx 3.4^{332}$ and later $\approx 14 k_{\mathrm{B}} T,{ }^{333}$ depending on concentrations of mono- and divalent cations. The ionic conditions dramatically influence also the forces required to disrupt the NCP-NCP contacts in chromatin fibers (typically $\sim 2-5 \mathrm{pN})$ and to induce DNA unwrapping from NCPs $(\sim 6-20 \mathrm{pN})$. These cohesive energies ensure fiber stability, but at the same time allow for some "unwrapping plasticity",334 and "breathing dynamics" of complexed NCPs, ${ }^{335}$ both required for DNA transcription to take place in the fiber. ${ }^{336-340}$

For top-to-bottom NCP-NCP contacts, the interactions are not ES and likely hydrophobic, providing the stability of NCP columns that are formed in solutions even at high [salt] and in different buffers. ${ }^{341}$ This NCP-NCP stacking governs the formation of NCP columns also in NCP crystals, ${ }^{342}$ semicrystalline NCP phases, ${ }^{343}$ as well as in NCP arcs and multilayered helices. ${ }^{344}$ The side-to-side contacts, with the wrapped DNA duplexes being often in close contact, on the contrary are likely ES in nature, see Fig. 22. For instance, close 
similarities in condensation and re-solubilization of DNA molecules and NCPs in the presence of multivalent cations support this statement. These [salt]-dependent ES contacts control the richness of mesophases formed in vitro by isolated NCPs in solutions at different [salt]. ${ }^{345}$

The presence of 8 highly basic Arg/Lys-rich flexible $\mathrm{N}$-terminal histone tails was associated with the NCP aggregation via "tail-bridging" effect in some experiments, ${ }^{346}$ computer simulations, ${ }^{347-349}$ and theoretical models. ${ }^{310,350}$ Tails-mediated NCP-NCP attraction, ${ }^{351}$ present at elevated [salt] only, seems however to be not very specific with respect to mutual NCPNCP orientations and as such is not likely to generate highlystructured chromatin fibers. Conversely, the close-range DNA-mediated ES side-to-side NCP-NCP contacts might be capable of doing so.

The stability of a number of dense NCP phases formed in vitro (inverse hexagonal, lamella-columnar, columnar hexagonal, etc.), see Fig. 3 in ref. 352, is modulated by NCP-NCP interactions. In particular, the formation NCP lamella-like bilayers of NCP columns ${ }^{341}$ at $3-30 \mathrm{mM}$ of $\mathrm{NaCl}$ and moderate 3-25 atm of external PEG pressure, is dictated by side-to-side DNA-mediated NCP-NCP contacts. DNAs on contacting NCPs are separated by only $5-15 \AA$ of water, that is within one Debye length. NCPs are oriented in NCP columns so that their dyad axes point on average perpendicular to the bilayer plane and the NCP sides with 2 DNA turns are hidden inside the bilayer. The NCP dyad orientations are distributed however within $\pm \approx 35^{\circ}$ from this preferred direction. ${ }^{341}$ On the contrary, the NCPs in non-interacting NCP columns in solution assume arbitrary azimuthal orientations. Stronger contacts of NCP sides with two turns of the wrapped DNA inside the bilayer govern the bilayer formation and are likely to dictate the peculiar azimuthal NCP frustrations observed in bilayers. $^{341}$

\section{NCP-NCP ES interactions and salt effects}

Wrapped DNA duplexes render the side-to-side NCP-NCP ES forces strongly azimuthally anisotropic. Crystal structures of NCPs exhibit close to 8 full DNA helical turns per one superhelical turn of DNA winding. DNA positive/negative charge zipper along the NCP-NCP side-to-side contact modulates the inter-NCP azimuthal interactions with a period of $\approx \pi / 4$, see Fig. 23. Note that DNA turns in NCPs are separated by the same "magic" $\approx 25-28 \AA$, where parallel DNA duplexes attract each other in dense assemblies with $\mathrm{MnCl}_{2}$, and 2 wrapped DNA turns bear a strong register in the NCP. ${ }^{353}$
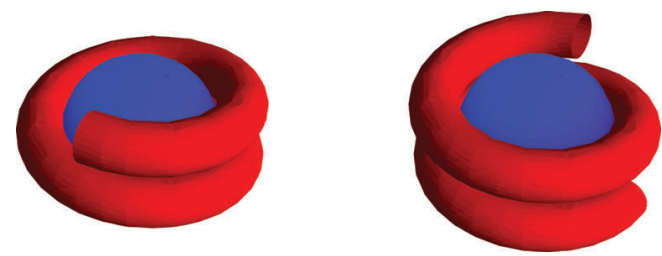

Fig. 22 Schematics of inter-nucleosomal interactions, with positive histone cores shown in blue and super-helically wrapped DNA strand depicted in red. The orientation of NCPs corresponds to a $2: 2$ DNA-DNA contact.
For a particular case of NCPs with parallel axes, it has been demonstrated that this azimuthal dependence of side-to-side $\mathrm{NCP}-\mathrm{NCP}$ contacts gives rise to a quantization of possible NCP orientations in nucleosomal bilayers, with the periodicity of $\approx 45^{\circ} .{ }^{354}$ The azimuthal optimization of side-to-side contacts of neighbouring NCPs in NCP bilayers resembles an azimuthal adaptation of short DNA fragments in columns of nano-DNAs in DNA cholesteric phases from section $5 .{ }^{182}$ Both effects originate from helix-specific DNA-DNA ES interactions.

Roughly speaking, the DNA contribution to NCP-NCP ES interaction energy is 4 times stronger for the NCP sides with 2 DNA turns than for 1-DNA-turn sides. For 2 NCPs with parallel axes, one can evaluate the ES forces and attractionrepulsion phase diagram, see Fig. 24. We have implemented a simple model with the ES double-layer repulsion for spherical histone cores (assumed to be uniformly charged sphere with the charge $Q$ ). For DNA part, the Derjaguin approximation was used for computing ES forces acting between bent DNA double helices, locally interacting according to eqn (2).

For a typical histone charge $Q=+220 e_{0}$, in physiological salt conditions, the model predicts for $2: 2$ DNA-DNA contacts the maximal NCP-NCP attractive forces of $2 \mathrm{pN}$ for $\theta=0.8, f=0.3$ (typical parameters used in DNA-DNA ES theory, as in Fig. 24). The NCP-NCP attraction reaches $8 \mathrm{pN}$ at $\theta=0.9, f=0.3$, and even $60 \mathrm{pN}$ at $\theta=0.8, f=0$ (stronger DNA-DNA attraction due to binding of cations into the major groove ${ }^{76}$ ). As the histone positive charge grows, the DH repulsion of NCP cores overwhelms DNA-DNA ES attraction. Thus, at larger $Q / e_{0}$ values, the NCP-NCP attraction region at $25-35 \AA$ between DNAs along the NCP-NCP contact disappears, see the inset in Fig. 24.

Helix-specific DNA-mediated ES attractions of 2:2 vs. $1: 1$ sides of NCPs are likely to cause NCP bilayer formation and NCP azimuthal frustrations. One cannot however exclude the possibility that histone tails bridge neighboring NCPs in NCP bilayers in azimuthally dependent manner, as suggested in ref. 341. In typical conditions, the range of DNA-mediated and tails-mediated NCP-NCP ES interactions might overlap, thus being hard to distinguish. Histone tails also follow the symmetry of DNA in NCPs, protruding into solution through the aligned DNA minor grooves of DNA super-helix.

In NCP crystals, the NCPs are densely packed, with the neighboring particles often contacting side-to-side by their

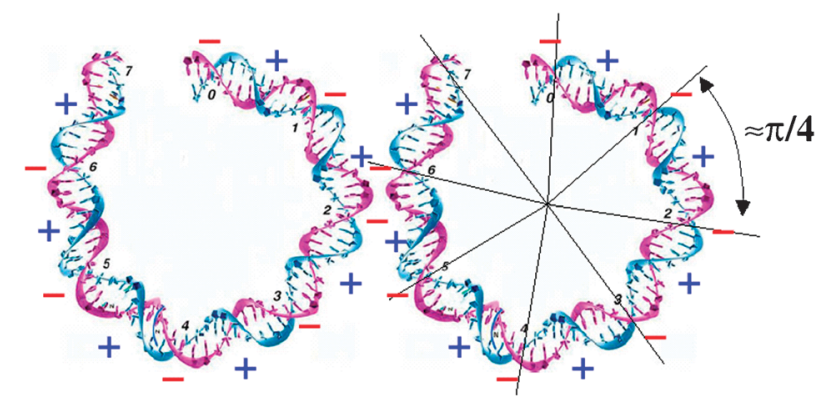

Fig. 23 A model for NCP-NCP interactions modulated by charge periodicity of the wrapped DNA. Zipper-like charge motif is viewed along the DNA superhelical axis, for prevalent counterion adsorption into the DNA major groove. The data of NCP laoi.pdb structure was used. The image is reproduced from ref. 354 with permission of IOP. 


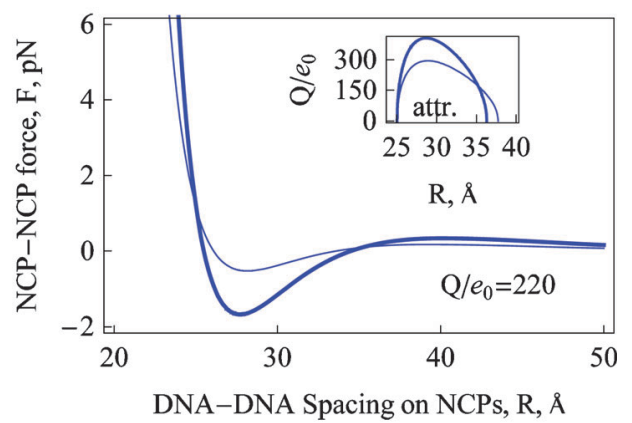

Fig. 24 ES force between two NCPs with parallel axes, for a histone charge of $+220 e_{0}$. Optimal NCP azimuthal alignment is assumed and $R$ is the separation between DNA axes on two NCPs contacting side-to-side ( $R=2 a=18 \AA$ corresponds to DNA-DNA contact). Thick and thin curves correspond to $2: 2$ and 1:1 DNA-DNA contacts, respectively. The ES NCP-NCP attraction-repulsion diagram is shown in the insert. The values for DNA parameters are: $\theta=0.8$, $f=0.3,1 / \kappa=7 \AA$, consistently through the paper.

DNAs. DNA-mediated interactions therefore have a profound effect on NCP crystal structure. It might differ strongly from one species to another, ${ }^{331}$ being a sensitive function of the crystallization buffer. For instance, the NCP crystals of yeast reveal an alternating pattern of $2: 2$ and $1: 1$ DNA-DNA contacts along the packed NCP columns, similarly to the case of NCP bilayers. The symmetry of frog's NCP crystals is different, without distinct NCP columns being formed, see ref. 331.

$\mathrm{MnCl}_{2}$ appears essential for NCP crystallization, being present at $\approx 30-70 \mathrm{mM}$ in crystallization buffers ${ }^{5,355}$ with $\mathrm{Mn}^{2+}$ cations occupying well-defined positions in NCP crystals. ${ }^{356}$ These cations stabilize histone-histone contacts: e.g. in yeast NCP crystals they are believed to be one of major driving forces for the crystal formation. ${ }^{331}$ In NCP crystals of other species, $\mathrm{Mn}^{2+}$ might hardly be involved in histone-histone contacts, such as NCP crystals of frog ${ }^{5}$ or human. ${ }^{357}$ The NCP crystals for all these 3 species however contain 5-10 $\mathrm{Mn}^{2+}$ per NCP that are associated with the DNA, see the green spheres in Fig. 16d. As we already know from section 2, strong $\mathrm{Mn}^{2+}$ binding to DNA grooves has a profound impact onto ES DNA-DNA forces that, in turn, might tune the formation of stable NCP crystals via NCP-NCP DNA-mediated side-side contacts.

\section{Implications for the fiber structure}

To reconcile numerous observations for semi-dense NCP phases, NCP crystals, and chromatin fibers, a rigorous theory of ES NCP-NCP interactions has to be developed. ${ }^{358}$ For arbitrary orientations of NCPs in space, it should take into account the helicity of DNA charges, a heterogeneous ${ }^{359}$ distribution of histone charges on the side and top/bottom NCP surfaces, a low-dielectric core of histones and DNA, ${ }^{360,361}$ a screening of coulombic interactions by electrolyte, and counterion condensation on NCPs. All this makes the development of such theory a frontier problem of mathematical physics, even on the level of the linear PB theory. Some aspects of it might be similar to the assembly of patchy colloidal spheres. ${ }^{362}$

With this NCP-NCP ES potential available, one can analyze the energetics, structure, and packing density of NCPs regularly arranged into chromatin fibers of different geometries. Being modulated by salt-dependent interactions, the chromatin fibers of different geometries might be stable in different salt buffers. Predictive power of such theory should however not be over-estimated. The geometrical wedge-shape of $\mathrm{NCPs}^{344,363}$ and interaction-mediated ${ }^{364}$ constraints anticipated for isolated NCPs will interfere with constraints of continuous DNA "wrapping" through the fiber structure. Looping and bending of linker DNAs ${ }^{365}$ influenced by binding of linker $\mathrm{H} 1 / \mathrm{H} 5$ histones is another stabilization mechanisms of a particular forms of "30 nm" chromatin fibers.

With all these complications for analytical modelling in mind, extensive computer simulations of fiber formation performed in recent years, ${ }^{366-370}$ provide a comprehensive complementary coverage on several intricate issues. With a growing computer power, nowadays, more fine and important details of NCP structure can be incorporated in largescale fiber simulations, rendering them more instructive and probably superior to analytical, "model" calculations with their limited applicability regimes.

\section{DNA compaction inside viruses and assembly of viral capsids}

Viral shells are composed via organized self-assembly of capsid proteins. One or several types of protein units are involved in this process, assembling into pentamers and hexamers, to form natural one-layer-thick (about $1-4 \mathrm{~nm}$ ) protein nano-containers that protect viral genomes. Often icosahedrally-shaped spherical viruses range about $20-100 \mathrm{~nm}$ in size, with prevailing ss-RNA viruses. Physical principles of capsomers' organization into these assemblies as well as the dynamical aspects of it are currently under intense investigation from experimental, simulational, and theoretical perspective. One source of information about the mechanical stability of viruses stems from recently developed nano-indentation technique, see Ref. 450. It allows to discriminate the contributions into the elastic modulus from the protein shell and nucleic acids packed inside it. Particularly for ds-DNA viruses, the ionic effects (mono- vs. DNA-condensing multi-valent cations) on the virion stability, known to modulate the strength of proteinprotein contacts and compressibility of densely-compacted viral genome, need a deeper physical understanding.

\section{A DNA packaging}

ES interactions of nucleic acids and capsid proteins are of primary importance for DNA/RNA compaction inside viruses and formation viral capsid shells. Many ds-DNA bacteriophages pack their DNA in amazingly dense and well-organized fashion. ${ }^{371-374}$ DNA densities can reach DNA-DNA distances of $R \approx 23-28 \AA$ creating the osmotic pressures of up to $\sim 50 \mathrm{~atm}$ inside them. ${ }^{375-377}$ Bacteriophages use these high pressures to inject their DNA into the cell cytoplasm upon infection. At such DNA packing densities, the effects of DNA helical structure are going to be pronounced in DNA-DNA ES forces, see Fig. 2.

Although the structures of viral capsids are well resolved in many $\mathrm{x}$-ray and cryo-EM studies, ${ }^{378-381}$ the precise packing arrangement of DNA inside them is often poorly known. 
Typical for ds-DNA viruses are the concentric rings of DNA, ${ }^{382-385}$ with DNA layers that are closer to the viral shell being better resolved by the cryo-EM image reconstruction, see Fig. 25. It corresponds to a co-axial inverse-spool model of DNA packing, with the outer (more ordered) shells of DNA spool being filled first. Average DNA-DNA separations in viruses increase when shorter DNAs are being packed inside, due to DNA-DNA repulsive ES and entropic forces. Recently, oriented DNA toroids condensed with spermine ${ }^{4+}$ inside T4 bacterio-phages ${ }^{386}$ and DNA "domain-wall" transitions upon DNA ejection from T5 phages ${ }^{387}$ have been clearly visualized by cryo-EM imaging.

As the capsids of many (but not all) ds-DNA viruses are penetrable for small ions, ${ }^{59}$ the presence of di- and tri-valent cations in solution can render the DNA-DNA ES forces inside the capsids more attractive. This will ease DNA packaging into and inhibit DNA ejection from such viruses. The latter fact can be a promising mechanism to "lock" the genome inside a capsid reducing the infective power of a particular ds-DNA virus. Indeed, only $1 \mathrm{mM}$ of spermine ${ }^{4+}$ in a buffer blocks nearly $90 \%$ of DNA inside $\lambda$-phage capsids. ${ }^{388}$ External osmotic pressure can also inhibit DNA release from the capsid. ${ }^{389}$

Several models of DNA compaction inside ds-DNA viruses have been developed in recent theoretical ${ }^{390-393}$ and computer simulation studies, ${ }^{394-396}$ including treatments of mechanical and DNA-DNA ES energies. To parameterize DNA-DNA repulsions at 5-25 $\AA$ between the surfaces, a simple exponential function has been typically used to fit the magnitude and decay length of DNA-DNA forces, as measured in dense columnar DNA assemblies. ${ }^{47}$ Note also recent theoretical ${ }^{397,398}$ and computer simulation ${ }^{399-401}$ advances that uncover the principles of biopolymer organization upon confinement in applications to DNA packing inside viral shells.

\section{B Capsid self-assembly and shell elasticity}

The self-assembly of viral capsomers, driven primarily by a burial of proteins' hydrophobic surfaces, ${ }^{402,403}$ typically yields mono-disperse viral shells. Some DNA-based viruses however assemble into polymorphic structures (e.g., polyoma- and HBV-virus), with transitions between different forms triggered by the $\mathrm{pH}$ level and some divalent cations thought to stabilize the ES contacts between capsid proteins. ${ }^{404-406}$ Capsids with different morphologies (for polyomavirus, e.g., icosahedral, octahedral, and tubular shapes) are realized due to different possible stable contacts of capsomer proteins. The shells of other viruses (e.g. CCMV-virus) require proper levels of divalent cations for their stability: the capsids swell without $\mathrm{Ca}^{2+}$ and at elevated $\mathrm{pH}$ levels. ${ }^{407-409}$ Many ss-RNA viruses compact their genomes using strongly basic flexible protein arms on the inner side of capsid proteins that provoke adsorption of flexible ss-RNA genome. ${ }^{410-413}$ All these facts underline the importance of ES forces for DNA/RNA packaging and capsid self-assembly, producing a growing number of theoretical studies on this hot topic in the last years. ${ }^{414-419}$

A number of theoretical ${ }^{420-422}$ and computational ${ }^{423-426}$ models exist for the capsid self-assembly. Some theories focus on the competition of attractive hydrophobic and repulsive ES interactions ${ }^{427}$ acting on a homogeneous capsid surface. Experimentally, some strengthening of inter-capsomer cohesive interactions has indeed been observed for the assembly of HBV shells at higher concentrations of simple salt, due to a stronger screening of ES capsomer-capsomer repulsion. ${ }^{403}$ Intrinsic curvature of capsomer-capsomer junctions predisposes the final size of capsids to be formed, while a flexibility of these connections permits the formation of shells of different sizes, e.g. for in vitro capsid assembly around artificial cores ${ }^{428,429}$ and polymers. $^{398}$

Capsomers of many viruses bear rather high electric charges on their surfaces and contain ionizable groups, ${ }^{430}$ that are often distributed quite non-uniformly, see Fig. 26. The effects of charge patchiness on capsomer assembly, shell morphology, and shells' mechanical properties have however not yet been studied theoretically. The inter-capsomer ES forces emerge via interaction of positively (Arg, Lys) and negatively (Asp, Glu) charged residues along the contact of protein sub-units. Shortranged ES contacts (salt bridges) can occur as well for amino acids separated by less than $3-5 \AA .431$

The elastic response of viral capsids has been investigated in recent experimental, ${ }^{432}$ several theoretical, ${ }^{43-436}$ and computer simulation $^{437-440}$ studies. The Young moduli for a number of DNA phages $(\lambda, \phi 29$, etc. $)$ were measured by a groundbreaking AFM nano-indentation technique, ${ }^{441}$ with elastic constants of up to $\sim 1.8 \mathrm{GPa}$ detected ${ }^{442}$ close to that of hard plastics. A bimodal distribution of the elastic constants identified for phi29 phage has been attributed to weaker protein-protein contacts in the equatorial regions of the shell. It indicates
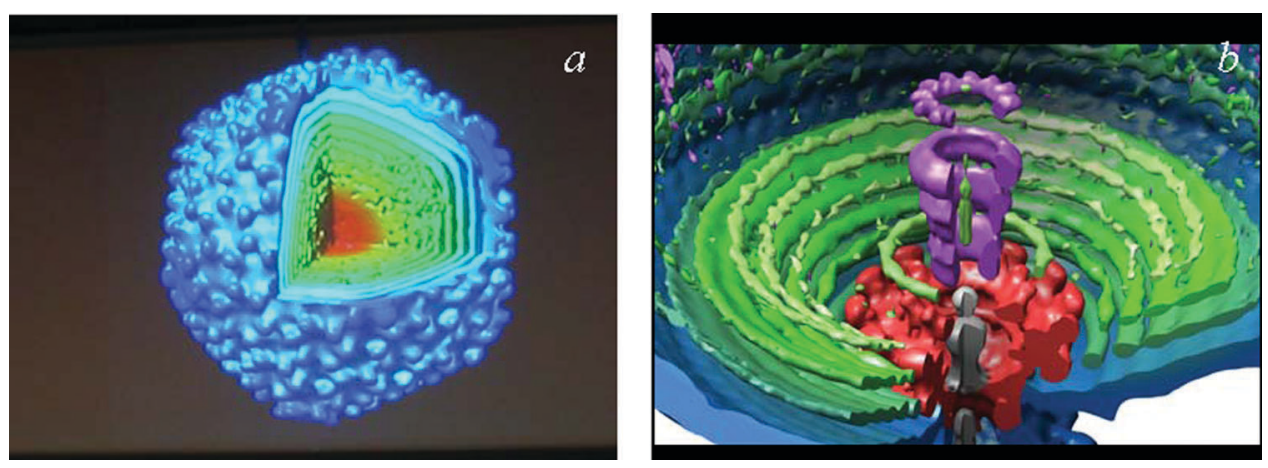

Fig. 25 The result of 3D reconstruction of cryo-EM images with the DNA layers visible inside P22 virus (a), with DNA layers being more ordered near the portal region (b, bottom). Images are the gift from Dr J. Johnson. 

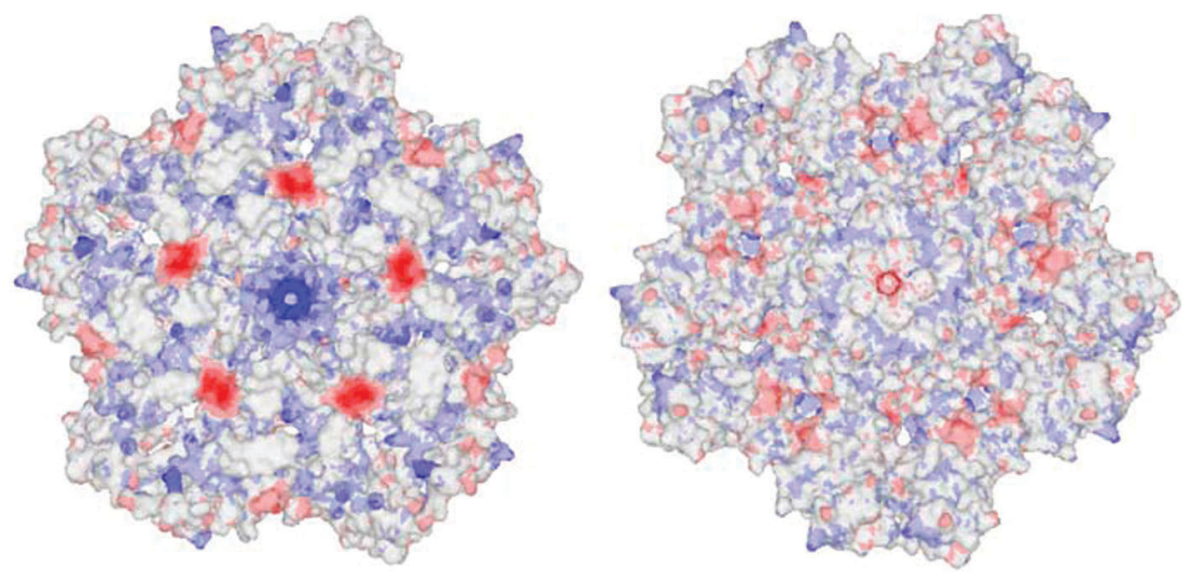

Fig. 26 Distribution of the ES potential (positive in blue and negative in red) on a pentameric unit of CCMV virus. The views shown are from the inside and outside of the capsid. The structure is visualized with MDL Chime and Protein Explorer using 1cwp.pdb entry.

non-completely equivalent ${ }^{443}$ interaction environments for capsomers on the shell surface. Another plausible explanation is that these two distinct values of elastic constants can reflect a different response of capsomer contacts for different pentamer and hexamer contacts realized in the capsid structure. Upon addition of $\mathrm{Mg}^{2+}$, the spring constant of DNA-filled $\lambda$-phage capsids was shown to drop considerably, ${ }^{444}$ indicating a reduced DNA-DNA mutual repulsion or cationic effects onto inter-capsomer ES contacts. ${ }^{445}$

For some ss-RNA viruses, the Young moduli are much smaller, e.g. $\approx 170 \mathrm{MPa}$ for CCMV-virus. ${ }^{446}$ The capsids of ss-RNA viruses do not need to sustain high pressures to protect their genomes inside, like ds-DNA bacteriophages do, with the doubly-charged ds-DNA inside that is in addition much less bendable and often densely packed. Swollen CCMV capsids, obtained at high $\mathrm{pH}$ levels and with no divalent cations, are even softer than that, see ref. 450. ss-RNA viruses typically sustain appreciably smaller AFM cantilever forces before breaking, as compared to ds-DNA bacteriophages, about 1 vs. $3 \mathrm{nN}$. To reconcile these observations and to provide a comparative analysis for different viruses, e.g., ds-DNA phages vs. ss-DNA viruses, a detailed molecular model is required that would couple the features of individual capsomer-capsomer contacts to capsid elastic constants and material properties. A tendency has been found in nano-indentation studies that capsids with extensive protein contacts (phages lambda and phi29) give rise to much more rigid shells, as compared to loosely assembled capsomers on the shells of relatively soft capsids (CCMV and HBV viruses). On the other hand, sometimes capsids of similar size and structure might exhibit a severalfold difference in their force-indentation response ${ }^{450}$.

\section{Perspectives and outlook}

In future, we plan to implement the DNA-DNA continuum ES theory from section 2 for the packing problems of semiflexible DNA-like PE chains in small cavities inside the viral shells. The effects of the varying [salt], specific DNA-condensing cations, different DNA lengths and capsid sizes are going to be considered. The underlying theory ${ }^{75,81}$ is to be modified via proper boundary conditions to describe a "deformed toroid" conformation of spooling DNAs. The growth mechanism of
DNA spool inside the capsids of different shapes, DNA-mediated ES repulsive pressures accumulated during this process, and the forces imposed on DNA upon its ejection ${ }^{377}$ can be evaluated. Cholesteric ES effects onto DNA packing properties can also be studied, section 5. For all these processes, at the DNA packing densities relevant for ds-DNA-viruses, the inherent DNA helical structure is going to have a large impact. At gigantic [DNA] maintained in many ds-DNA viruses, the cation-specific ES forces, rather than hydration forces as suggested in some recent exprimental studies ${ }^{444,445}$, are likely to dominate the DNA-DNA interactions, see Fig. 2. The dynamical aspects of DNA packaging, such as the time of DNA release from the capsid $^{447}$ through a thin channel ${ }^{448,449}$ and pauses in DNA packing/ejection caused by necessary rearrangements of DNA spool $^{399}$ are another hot topics of the research.

The organization of flexible ss-RNAs inside viruses with non-uniformly charged capsid interior is also important to understand. The landscape of positive residues on the inner capsid surface can be extracted from PDB data of the capsomers. They interact via screened Coulomb potentials with flexible ss-RNA chains inside the shell. Here, our theory of PE adsorption onto oppositely charged obstacles ${ }^{451-453}$ can be modified to incorporate the charge patchiness ${ }^{454,455}$ and a concave shape of adsorbing surfaces. The outcomes of such an extension for the thickness of adsorbed PE layers and critical adsorption conditions can be compared with experimental data available for ss-RNA layering profiles close to viral shells ${ }^{410}$ and for the charge stoichiometry of (basic tails) vs. (negative ss-RNA). ${ }^{411}$

A detailed analysis of inter-capsomer ES interactions can be performed, disregarded in many coarse-grained models of capsid self-assembly. The details of charge patterns on nano-scale along the contact of capsomers can be extracted from their PDB/VDB entries, similarly to analysis of DNA-protein complexes in section 9c. For different viruses, the ES interactions of capsomers and energetic costs of deformations of inter-capsomer connections (for in-plane rotation and out-of plane bending of pentamers and hexamers) can then be enumerated and parameterized. The strength of ES adhesion and hydrophobic contacts of capsomers might be compared with the general tendencies of (strongly overestimated ${ }^{456}$ ) capsomercapsomer affinities listed in the VDB. 
Inter-capsomer interaction potentials will be used to examine capsomers' optimal arrangements on the shell surfaces for different symmetries of subunit positioning. One can start with a phenomenological model of capsid self-assembly, with azimuthally-dependent inter-capsomer potentials and elastic contacts. With more realistic energy parameterization based on molecular structure data, one can revise numerically the capsid self-assembly for different shell morphologies (spherical, icosahedral, octahedral) and environmental conditions ([salt], pH). Optimal capsomer arrangements follow from minimization of the sum of interaction and deformation energies. The elastic penalties of dihedral out-of-plane deformations and azimuthal in-plane capsomer rotations are to be accounted for. Sampling of assembled conformations can hopefully produce an optimal viral structure.

The elastic properties of empty shells can later be addressed by the theory, both for highly and weakly charged shells. One can anticipate for instance smaller elastic constants for the shells with pronounced polymorphic behavior. The impact of tightly packed ds-DNA and loosely confined ss-RNA onto elastic stiffness of full virions upon their squeezing is important to tackle as well. Intuitively, capsids with a larger electric charge have to be harder to bend and buckle. All these speculations remain to be validated by theoretical and computational modelling, ${ }^{457-460}$ and probably checked in future experiments.

One technique for a comparative analysis of protein-protein contacts is mutation experiments. A number of experimental studies revealed that a substitution of amino acids along the contact of capsomer proteins can impair the infectivity of some viruses, ${ }^{461,462}$ including destabilizing mutations. Interactions of amino acids across such chemically modified capsomers' interfaces often involve a tunable ES component. $^{463,464}$ Understanding of its implications is important for rational design of viral capsids with particular physico-chemical properties, used in modern medicine as nano-containers for drug and gene delivery purposes.

\section{Conclusions}

In this perspective, we focused on recent developments and new viewpoints on ES effects for a number of biological DNA-related systems. Several experimental achievements and DNA-related phenomena discovered in the last years are overviewed, which challenge theoretical and computational modelling. In the course of presentation, as the systems become more complex, it is progressively harder to unravel the physical principles behind their functioning. Some analytical insights from our recent developments are discussed, that uncover the general principles behind charge-mediated DNA-DNA, DNA-protein, and NCP-NCP interactions. The PE models of DNA and statistical analysis of structure information on proteins have also been applied to some nanotechnology applications, principles of bio-molecular DNA-protein recognition, and self-assembly.

Despite inherent limitations of mean-field PB-like theories of DNA, the biophysical approaches developed often enabled us to rationalize the system's structural properties as dictated by intermolecular forces. The conceptual frameworks proposed in the paper allowed us to anticipate the physical effects in DNA-related systems that are still too large for modern ab initio computer simulations. Clearly, more work is to be done to achieve a quantitative understanding of all these complex phenomena. In particular, the behavior of interconnected NCPs in chromatin fibers, DNA packaging in phage heads, and self-assembly of viral shells feature lots of important biological details to be incorporated in future theoretical models.

$\begin{array}{ll}\text { Abbreviations } \\ \text { ES } & \text { electrostatic } \\ \text { HB } & \text { hydrogen bond } \\ \text { PE } & \text { polyelectrolyte } \\ \text { PB } & \text { Poisson-Boltzmann } \\ \text { PEG } & \text { polyethylene glycol } \\ \text { DH } & \text { Debye-Hückel } \\ \text { EM } & \text { electron microscopy } \\ \text { AFM } & \text { atomic force microscopy } \\ \text { bp } & \text { base pair } \\ \text { kbp } & \text { kilo base pair } \\ \text { [DNA] } & \text { DNA concentration } \\ \text { [salt] } & \text { salt concentration } \\ \text { ds } & \text { double stranded } \\ \text { ss } & \text { single stranded } \\ \text { CL } & \text { cationic lipids } \\ \text { LC } & \text { liquid-crystalline } \\ \text { GNP } & \text { gold nano-particle } \\ \text { hom } & \text { homologous } \\ \text { NCP } & \text { nucleosome core particle } \\ \text { PDB } & \text { Protein Data Bank } \\ \text { VDB } & \text { Viper Data Bank } \\ & \end{array}$

\section{Acknowledgements}

This article is based on the results of collaboration and joint publications with R. Everaers (ENS, Lyon, France), A. Kornyshev (Imperial College, London, UK), A. Kolomeisky (Rice University, TX, US), S. Leikin (NIH, MD, US), A. Poghossian (IBN-2, FZ Jülich, Germany), and R. Winkler (ICS-2, FZ Jülich, Germany). I am very grateful to them for stimulating discussions and encouragement. I am also thankful to T. Bellini, D. Lee, N. Kleckner, W. Olson, E. Petrov, M. Peyrard, M. Prentiss, E. Starostin, A. Travers, G. Wuite, A. Zlotnick and $\mathrm{G}$. Zanchetta for scientific discussions and correspondence. I acknowledge financial support from the German Research Foundation, DFG.

\section{References}

1 W. M. Gelbart, et al., Phys. Today, 2000, 53, 38.

2 S. McLaughlin, Annu. Rev. Biophys. Biophys. Chem., 1989, 18, 113.

3 C. G. Kalodimos, et al., Science, 2004, 305, 386.

4 B. Honig and A. Nicholls, Science, 1995, 268, 1144

5 K. Luger, et al., Nature, 1997, 389, 251.

6 T. J. Richmond and C. Davey, Nature, 2003, 423, 145.

7 J. H. Morais-Cabral, et al., Nature, 2001, 414, 37.

8 M. S. Weiss, et al., Science, 1991, 254, 1627. 
9 G. C. L. Wong and L. Pollack, Annu. Rev. Phys. Chem., 2010, 61, 171.

10 A. Ciudad, J. M. Sancho and G. P. Tsironis, J. Biol. Phys., 2006, 32455.

11 A. A. Kornyshev, D. J. Lee, S. Leikin and A. Wynveen, Rev. Mod. Phys., 2007, 79, 943.

12 S. J. Chen, Annu. Rev. Biophys., 2008, 37, 197.

13 A. Warshel and T. S. Russell, Q. Rev. Biophys., 1984, 17, 3.

14 K. A. Sharp and B. Honig, Annu. Rev. Biophys. Biophys. Chem., 1990, 19, 301.

15 M. E. Davis and J. A. McCammon, Chem. Rev., 1990, 90, 509.

16 F. B. Sheinerman, R. Norel and B. Honig, Curr. Opin. Struct. Biol., 2000, 10, 153.

17 A. H. Elcock, et al., J. Phys. Chem. B, 2001, 105, 1504

18 A. Warshel, et al., Biochim. Biophys. Acta, Proteins Proteomics, 2006, 1764, 1647.

19 R. P. Rand, Annu. Rev. Biophys. Bioeng., 1981, 10, 277.

20 G. Cevc, Biochim. Biophys. Acta, 1990, 1031, 311.

21 R. Messina, J. Phys.: Condens. Matter, 2009, 21, 113102

22 P. H. von Hippel, Science, 2004, 305, 350.

23 A. J. McCoy, et al., J. Mol. Biol., 1997, 268, 570.

24 H. Schiessel, J. Phys.: Condens. Matter, 2003, 15, R699.

25 C. L. Woodcock and R. P. Ghosh, Cold Spring Harbor Perspect. Biol., 2010, 2, a000596.

26 C. M. Knobler and W. M. Gelbart, Annu. Rev. Phys. Chem., 2009, 60, 367.

27 M. M. A. E. Claessens, et al., Proc. Natl. Acad. Sci. U. S. A., 2008, 105, 8819 .

28 D. E. Draper, Biophys. J., 2008, 95, 5489.

29 D. A. Doyle, et al., Science, 1998, 280, 69.

30 N. A. Baker, et al., Proc. Natl. Acad. Sci. U. S. A., 2001, 98, 10037.

31 R. D. Kornberg, et al., Science, 2001, 292, 1863.

32 V. A. Parsegian and D. Gingell, J. Adhes., 1972, 4, 283.

33 S. Leikin, V. A. Parsegian and D. C. Rau, Annu. Rev. Phys. Chem., 1993, 44, 369.

34 R. H. French, et al., Rev. Mod. Phys., 2010, 82, 1887.

35 G. S. Manning, Q. Rev. Biophys., 1978, 11, 179.

36 G. S. Manning, Macromolecules, 2001, 34, 4650.

37 G. S. Manning, J. Phys. Chem. B, 2007, 111, 8554.

38 R. M. M. Smeets, Nano Lett., 2006, 6, 89.

39 U. F. Keyser, et al., Nat. Phys., 2006, 2, 473.

40 S. van Dorp, et al., Nat. Phys., 2009, 5, 347.

41 A. G. Cherstvy, J. Chem. Phys., 2005, 123, 116101.

42 J. DeRouchey, V. A. Parsegian and D. C. Rau, Biophys. J., 2010, 99, 2608.

43 D. C. Rau and V. A. Parsegian, Science, 1990, 249, 1278.

44 S. Leikin, D. C. Rau and V. A. Parsegian, Proc. Natl. Acad. Sci. U. S. A., 1994, 91, 276.

45 V. A. Parsegian, N. Fuller and R. P. Rand, Proc. Natl. Acad. Sci. U. S. A., 1979, 76, 2750.

46 S. Leikin, D. C. Rau and V. A. Parsegian, Phys. Rev. A: At., Mol., Opt. Phys., 1991, 44, 5272.

47 D. C. Rau and V. A. Parsegian, Biophys. J., 1992, 61, 260; D. C. Rau and V. A. Parsegian, Biophys. J., 1992, 61, 272.

48 B. A. Todd, et al., Biophys. J., 2008, 94, 4775.

49 H. H. Strey, V. A. Parsegian and R. Podgornik, Phys. Rev. E: Stat. Phys., Plasmas, Fluids, Relat. Interdiscip. Top., 1999, 59, 999.

50 J. Widom and R. L. Baldwin, Biopolymers, 1983, 22, 1595.

51 V. A. Bloomfield, Biopolymers, 1991, 31, 1471.

52 L. C. Gosule and J. A. Schellman, Nature, 1976, 259, 333.

53 U. K. Laemmli, Proc. Natl. Acad. Sci. U. S. A., 1975, 72, 4288.

54 J. DeRouchey, R. R. Netz and J. O. Raedler, Eur. Phys. J. E, 2005, 16, 17.

55 X. Qiu, V. A. Parsegian and D. C. Rau, Proc. Natl. Acad. Sci. U. S. A., 2010, 107, 21482.

56 N. V. Hud, et al., Biochem. Biophys. Res. Commun., 1993, 193, 1347.

57 M. W. Hsiang and R. D. Cole, Proc. Natl. Acad. Sci. U. S. A., 1977, 72, 4288.

58 P. Shah and E. Swiatlo, Mol. Microbiol., 2008, 68, 4.

59 B. N. Ames and D. T. Dubin, J. Biol. Chem., 1960, 235, 769.

60 M. Kanduč, J. Dobnikar and R. Podgornik, Soft Matter, 2009, 5, 868.
61 M. Kanduč, A. Naji and R. Podgornik, J. Chem. Phys., 2010, 132, 224703.

62 V. B. Teif and K. Bohinc, Prog. Biophys. Mol. Biol., 2010, DOI: 10.1016/j.pbiomolbio.2010.07.002.

63 J. Landy and J. Rudnick, Phys. Rev. E: Stat., Nonlinear, Soft Matter Phys., 2010, 81, 061918.

64 I. Rouzina and V. A. Bloomfield, J. Phys. Chem., 1996, 100, 9977.

65 B. I. Shklovskii, Phys. Rev. Lett., 1999, 82, 3268.

66 H. Boroudjerdi, et al., Phys. Rep., 2005, 416, 129.

67 Y. Levin, Rep. Prog. Phys., 2002, 65, 1577.

68 N. Gronbech-Jensen, R. J. Mashl, R. F. Bruinsma and W. M. Gelbart, Phys. Rev. Lett., 1997, 78, 2477.

69 E. Allahyarov and H. Löwen, Phys. Rev. E: Stat. Phys., Plasmas, Fluids, Relat. Interdiscip. Top., 2000, 62, 5542.

70 E. Allahyarov, G. Gompper and H. Löwen, Phys. Rev. E: Stat., Nonlinear, Soft Matter Phys., 2004, 69, 041904.

71 A. Naji, A. Arnold, C. Holm and R. R. Netz, Europhys. Lett., 2004, 67, 130.

72 M. Deserno, A. Arnold and C. Holm, Macromolecules, 2003, 36, 249.

73 B. Luan and A. Aksimentiev, J. Am. Chem. Soc., 2008, 130, 15754.

74 K. Wagner, et al., Biophys. J., 1997, 73, 21.

75 A. A. Kornyshev and S. Leikin, J. Chem. Phys., 1997, 107, 3656.

76 A. A. Kornyshev and S. Leikin, Phys. Rev. Lett., 1999, 82, 4138.

77 H. H. Strey, V. A. Parsegian and R. Podgornik, Phys. Rev. Lett., 1997, 78, 895.

78 A. A. Kornyshev and S. Leikin, Proc. Natl. Acad. Sci. U. S. A., 1998, 95, 13579

79 W. K. Olson, et al., Proc. Natl. Acad. Sci. U. S. A., 1998, 95, 11163.

80 A. A. Kornyshev and S. Leikin, Phys. Rev. Lett., 2001, 86, 3666.

81 A. G. Cherstvy, A. A. Kornyshev and S. Leikin, J. Phys. Chem. B, 2002, 106, 13362.

82 A. G. Cherstvy, A. A. Kornyshev and S. Leikin, J. Phys. Chem. B, 2004, 108, 6508.

83 A. A. Kornyshev and A. Wynveen, Phys. Rev. E: Stat., Nonlinear, Soft Matter Phys., 2004, 69, 041905.

84 A. Wynveen, et al., Nucleic Acids Res., 2008, 36, 5540.

85 A. A. Kornyshev and S. Leikin, Phys. Rev. Lett., 2000, 84, 2537.

86 A. A. Kornyshev, S. Leikin and S. V. Malinin, Eur. Phys. J. E; Soft Matter Biol. Phys., 2002, 7, 83.

87 A. Wynveen, D. J. Lee and A. A. Kornyshev, Eur. Phys. J. E, $2005,16,303$.

88 H. M. Harreis, et al., Phys. Rev. Lett., 2002, 89, 018303.

89 A. A. Kornyshev, et al., Phys. Rev. Lett., 2005, 95, 148102.

90 H. H. Strey, et al., Phys. Rev. Lett., 2000, 84, 3105.

91 D. J. Lee, S. Leikin and A. Wynveen, J. Phys.: Condens. Matter, 2010, 22, 072202.

92 D. J. Lee, A. Wynveen, A. A. Kornyshev and S. Leikin, J. Phys. Chem. B, 2010, 114, 11668 .

93 D. J. Lee, J. Phys.: Condens. Matter, 2010, 22, 414101.

94 A. A. Kornyshev, Phys. Chem. Chem. Phys., 2010, 12, 12352.

95 V. Lorman, R. Podgornik and B. Žekš, Phys. Rev. Lett., 2001, 87, 218101.

96 G. M. Grason, Europhys. Lett., 2008, 83, 58003.

97 C. L. Ma and V. A. Bloomfield, Biophys. J., 1994, 67, 1678.

98 A. Warshel, M. Kato and A. V. Pisliakov, J. Chem. Theory Comput., 2007, 3, 2034

99 M. V. Fedorov and A. A. Kornyshev, Mol. Phys., 2007, 105, 1.

100 A. A. Kornyshev and G. Sutmann, J. Chem. Phys., 1996, 1524, 104.

101 S. Gavryushov, J. Phys. Chem. B, 2007, 111, 5264.

102 S. A. Pabit, et al., Nucleic Acids Res., 2009, 37, 3887.

103 T. E. Angelini, et al., Proc. Natl. Acad. Sci. U. S. A., 2003, 100, 8634.

104 F. Tombolato, A. Ferrarini and E. Grelet, Phys. Rev. Lett., 2006, 96, 258392.

105 V. A. Bloomfield, Curr. Opin. Struct. Biol., 1996, 6, 334.

106 S. Levin-Zaidman, Science, 2003, 299, 254.

107 J. Englander, et al., J. Bacteriol., 2004, 186, 5973.

108 N. V. Hud, et al., Biochem. Biophys. Res. Commun., 1993, 193, 1347.

109 A. Leforestier and F. Livolant, Proc. Natl. Acad. Sci. U. S. A., 2009, 106, 9157. 
110 N. V. Hud and K. N. Downing, Proc. Natl. Acad. Sci. U. S. A., 2001, 98, 14925 .

111 S. M. Douglas, et al., Nature, 2009, 459, 414.

112 H. Dietz, et al., Science, 2009, 325, 725.

113 A. G. Cherstvy, J. Phys.: Condens. Matter, 2005, 17, 1363.

114 C. G. Baumann, S. B. Smith, V. A. Bloomfield and C. Bustamante, Proc. Natl. Acad. Sci. U. S. A., 1997, 94, 6185.

115 J. P. Peters and L. J. Maher III, Q. Rev. Biophys., 2010, 43, 23.

116 E. Starostin and R. Everaers, PRE, 2010, submitted.

117 J. Ubbink and T. Odijk, Europhys. Lett., 1996, 33, 353.

118 J. Ubbink and T. Odijk, Biophys. J., 1995, 68, 54.

119 J. Kindt, et al., Proc. Natl. Acad. Sci. U. S. A., 2001, 98, 13671.

120 W. S. Klug and M. Ortiz, J. Mech. Phys. Solids, 2003, 51, 1815.

121 E. L. Starostin, J. Chem. Phys., 2008, 129, 154104.

122 I. M. Kulic, D. Andrienko and M. Deserno, Europhys. Lett., 2004, 67, 418.

123 N. V. Hud, et al., Proc. Natl. Acad. Sci. U. S. A., 1995, 92, 3581.

124 B. van den Broek, et al., Biophys. J., 2010, 98, 1902.

125 C. Battle, et al., Phys. Rev. E: Stat., Nonlinear, Soft Matter Phys., 2009, 80, 031917.

126 Y. Sh. Mamasakhlisov, et al., Phys. Rev. E: Stat., Nonlinear, Soft Matter Phys., 2009, 80, 031915.

127 G. G. Pereira and D. R. M. Williams, Biophys. J., 2001, 80, 161.

128 X. Zhang, et al., Chin. Sci. Bull., 2009, 54, 2425.

129 C. Herold, et al., Phys. Rev. Lett., 2010, 104, 148102.

130 E. Petrov, personal communication.

131 J. O. Raedler, I. Koltover, T. Salditt and C. R. Safinya, Science, 1997, 275, 810.

132 G. C. L. Wong, et al., Science, 2000, 288, 2035.

133 U. Raviv, et al., Proc. Natl. Acad. Sci. U. S. A., 2005, 102, 11167.

134 L. Tang, et al., Nat. Mater., 2004, 3, 615.

135 G. Martini and L. Ciani, Phys. Chem. Chem. Phys., 2009, 11, 211.

136 K. K. Ewert, et al., Expert Opin. Biol. Ther., 2005, 55, 33.

137 I. Koltover, T. Salditt, J. O. Raedler and C. R. Safinya, Science, 1998, 281, 78.

138 G. Caracciolo, et al., J. Phys. Chem. B, 2010, 114, 2028.

139 K. Wagner, et al., Langmuir, 2000, 16, 303.

140 D. Harries, S. May, W. M. Gelbart and A. Ben-Shaul, Biophys. J., 1998, 75, 159.

141 S. May, D. Harries and A. Ben-Shaul, Biophys. J., 2000, 78, 1681.

142 D. Harries, S. May and A. Ben-Shaul, J. Phys. Chem. B, 2003, 107, 3624 .

143 D. Harries, et al., Colloids Surf., A, 2002, 208, 41.

144 O. Farago, N. Gronbech-Jensen and P. Pincus, Phys. Rev. Lett., 2006, 96, 018102.

145 O. Farago and N. Cronbech-Jensen, Biophys. J., 2007, 92, 3228.

146 L. Gao, J. Cao and W. Fang, J. Phys. Chem. B, 2010, 114, 7261.

147 O. Farago and N. Cronbech-Jensen, J. Am. Chem. Soc., 2009, 131, 2875.

148 G. Tresset and Y. Lansac, J. Phys. Chem. Lett., 2011, 2, 41.

149 C. Fleck, R. R. Netz and H. H. von Grünberg, Biophys. J., 2002, 82, 76.

150 H. Schiessel and H. Aranda-Espinoza, Eur. Phys. J. E: Soft Matter Biol. Phys., 2001, 5, 499.

151 A. G. Cherstvy, J. Phys. Chem. B, 2007, 111, 12933.

152 I. Koltover, T. Salditt, J. O. Raedler and C. R. Safinya, Phys. Rev. Lett., 1997, 79, 2582.

153 T. Salditt, I. Koltover, J. O. Raedler and C. R. Safinya, Phys. Rev. E: Stat. Phys., Plasmas, Fluids, Relat. Interdiscip. Top., 1998, 58, 889.

154 R. Menes, N. Gronbech-Jensen and P. A. Pincus, Eur. Phys. J. E: Soft Matter Biol. Phys., 2000, 1, 345.

155 R. R. Netz, Phys. Rev. E: Stat. Phys., Plasmas, Fluids, Relat. Interdiscip. Top., 1999, 60, 3174.

156 I. Koltover, K. Wagner and C. R. Safinya, Proc. Natl. Acad. Sci. U. S. A., 2000, 97, 14046.

157 E. Raspaud, D. Durand and F. Livolant, Biophys. J., 2005, 88, 392.

158 Y. S. Tarahovsky, et al., Biophys. J., 2004, 87, 1054.

159 B. Ma, et al., J. Controlled Release, 2007, 123, 184.

160 P. Varnai and Y. Timsit, PLoS One, 2010, 5, e9326.

161 A. Leforestier and F. Livolant, Biophys. J., 1993, 65, 56.

162 T. E. Strzelecka, M. W. Davidson and R. L. Rill, Nature, 1988, 331, 457.

163 Y. M. Yevdokimov, et al., Liq. Cryst., 1988, 3, 1443.
164 A. Leforestier, et al., C. R. Chim., 2008, 11, 229.

165 I. W. Hamley, Soft Matter, 2010, 6, 1863.

166 A. D. Rey, Soft Matter, 2010, 6, 3402.

167 Z. Dogic and S. Fraden, Macromolecules, 2000, 16, 7820.

168 E. Grelet and S. Fraden, Phys. Rev. Lett., 2003, 90, 198302.

169 L. Rudd, D. J. Lee and A. A. Kornyshev, Phys. Chem. Chem. Phys., 2006, 8, 4347.

170 J. Lepault, et al., EMBO J., 1987, 6, 1507.

171 F. Livolant and M. F. Maestre, Biochemistry, 1988, 27, 3056.

172 N. S. Blanc, et al., J. Struct. Biol., 2001, 134, 76.

173 C. B. Stanley, H. Hong and H. H. Strey, Biophys. J., 2005, 89, 2552.

174 J. Pelta, D. Durand, J. Doucet and F. Livolant, Biophys. J., 1996, 71, 48.

175 A. A. Kornyshev and S. Leikin, Phys. Rev. E: Stat. Phys., Plasmas, Fluids, Relat. Interdiscip. Top., 2000, 62, 2576.

176 V. L. Golo, et al., Eur. Phys. J. E, 2010, 31, 59.

177 J. P. Straley, Phys. Rev. A: At., Mol., Opt. Phys., 1976, 14, 1835.

178 F. Tombolato and A. Ferrarini, J. Chem. Phys., 2005, 122, 054908

179 A. G. Cherstvy, J. Phys. Chem. B, 2008, 112, 12585.

180 F. G. Donnan, Chem. Rev., 1924, 1, 73.

181 D. J. Lee, et al., work in preparation.

182 G. Zanchetta, et al., Proc. Natl. Acad. Sci. U. S. A., 2010, 107, 17497.

183 M. Nakata, et al., Science, 2007, 318, 1276.

184 G. Zanchetta, et al., Proc. Natl. Acad. Sci. U. S. A., 2008, 105, 1111.

185 G. Zanchetta, et al., J. Phys.: Condens. Matter, 2008, 20, 494214.

186 P. Varnai and Y. Timsit, Nucleic Acids Res., 2010, 38, 4163.

187 A. Goldar, et al., J. Phys.: Condens. Matter, 2008, 20, 035102.

188 A. A. Kornyshev and A. Wynveen, Proc. Natl. Acad. Sci. U. S. A., 2009, 106, 4683.

189 D. J. Lee and A. A. Kornyshev, J. Chem. Phys., 2009, 131, 155104

190 A. Barzel and M. Kupiec, Nat. Rev. Genet., 2008, 9, 27.

191 A. Weiner, N. Zauberman and A. Minsky, Nat. Rev. Microbiol., 2009, 7, 748.

192 A. Falashi, HFSP J., 2008, $2,257$.

193 X. J. Lu and W. K. Olson, Nucleic Acids Res., 2003, 31, 5108.

194 W. K. Olson and V. B. Zhurkin, Curr. Opin. Struct. Biol., 2000, 10, 286.

195 A. A. Gorin, V. B. Zhurkin and W. K. Olson, J. Mol. Biol., 1995, 247, 34.

196 A. G. Cherstvy, PhD Thesis, Düsseldorf University, 2002.

197 B. M. Weiner and N. Kleckner, Cell, 1994, 77, 977.

198 S. M. Burgess, Adv. Genet., 2002, 46, 49.

199 S. Inoue, et al., Biochemistry, 2007, 46, 164.

200 G. S. Baldwin, et al., J. Phys. Chem. B, 2008, 112, 1060.

201 M. Prentiss, et al., Proc. Natl. Acad. Sci. U. S. A., 2009, 106, 19824.

202 M. Prentiss, personal communication.

203 Y. Saitoh and U. K. Laemmli, Cold Spring Harbor Sym. Quant. Biol., 1993, LVIII, 755.

204 N. Kleckner, Proc. Natl. Acad. Sci. U. S. A., 1996, 93, 8167.

205 A. G. Cherstvy, J. Mol. Recognit., 2011, 24, 283.

206 B. S. Singer, et al., Cell, 1982, 31, 25.

207 B. Bhushan, Philos. Trans. R. Soc. London, Ser. A, 2008, 388, 1499.

208 M. Urbakh and E. Meyer, Nat. Mater., 2010, 9, 8.

209 I. Barel, et al., Phys. Rev. Lett., 2010, 104, 066104.

210 N. Clauvelin, B. Audoly and S. Neukirch, Macromolecules, 2008, 41, 4479.

211 N. Clauvelin, et al., Biophys. J., 2009, 96, 3716.

212 R. T. Dame, et al., Nature, 2006, 444, 387.

213 M. C. Noom, et al., Nat. Methods, 2007, 4, 1031.

214 A. G. Cherstvy, J. Phys. Chem. B, 2009, 113, 5350.

215 D. Poland and H. A. Scheraga, Theory of Helix-Coil Transition in Biopolymers, Academic Press, New York, 1970.

216 M. Peyrard and A. R. Bishop, Phys. Rev. Lett., 1989, 62, 2755.

217 S. Ares, et al., Phys. Rev. Lett., 2005, 94, 035504.

218 D. Jost and R. Everaers, J. Phys.: Condens. Matter, 2009, 21, 034108.

219 D. Jost and R. Everaers, Biophys. J., 2009, 96, 1056. 
220 M. Joyeux and A. Florescu, J. Phys.: Condens. Matter, 2009, 21, 034101 .

221 S. Cuesta-Lopez, D. Angelov and M. Peyrard, Europhys. Lett., 2009, 87, 48009.

222 A. G. Cherstvy and A. A. Kornyshev, J. Phys. Chem. B, 2005, 109, 13024.

223 S. Chang, et al., J. Phys.: Conf. Ser., 2006, 29, 18.

224 D. Grasso, et al., Liq. Cryst., 1991, 9, 299.

225 R. Jin, et al., J. Am. Chem. Soc., 2003, 125, 1643.

226 H. Long, et al., J. Phys. Chem. B, 2006, 110, 2918.

227 O.-S. Lee, et al., J. Phys. Chem. Lett., 2010, 1, 1781.

228 I. Eryazici, et al., J. Am. Chem. Soc., 2010, 132, 17068.

229 C. Schildkraut and S. Lifson, Biopolymers, 1965, 3, 195.

230 M. Peyrard, et al., work in preparation, personal communication.

231 M. J. Schoning and A. Poghossian, Electroanalysis, 2006, 18, 1893.

232 A. G. Cherstvy, et al., Sensors \& Actuators B, submitted.

233 A. E. Prigodich, et al., J. Am. Chem. Soc., 2010, 132, 10638.

234 D. Irving, P. Gong and R. Levicky, J. Phys. Chem. B, 2010, 114, 7631.

235 A. Poghossian, et al., Sens. Actuators, B, 2005, 111-112, 470.

236 I. Y. Wong and N. A. Melosh, Nano Lett., 2009, 9, 3521.

237 Y. Choo and A. Klug, Curr. Opin. Struct. Biol., 1997, 7, 117.

238 J. A. McCammon, Curr. Opin. Struct. Biol., 1998, 8, 245.

239 R. B. Winter, O. G. Berg and P. H. von Hippel, Biochemistry, 1981, 20, 6961.

240 O. G. Berg, R. B. Winter and P. H. von Hippel, Biochemistry, 1981, 20, 6929.

241 S. E. Halford and J. F. Marko, Nucleic Acids Res., 2004, 32, 3040.

242 S. E. Halford, Biochem. Soc. Trans., 2009, 37, 343.

243 A. B. Kolomeisky, Phys. Chem. Chem. Phys., 2011, 13, 2088.

244 R. K. Das and A. B. Kolomeisky, Phys. Chem. Chem. Phys., 2010, 12, 2999.

245 A.-M. Florescu and M. Joyeux, J. Phys. Chem. A, 2010, 114, 9662.

246 Y. von Hansen, R. R. Netz and M. Hinczewski, J. Chem. Phys., 2010, 132, 135103.

247 M. T. Record, et al., Biochemistry, 1977, 16, 4791.

248 M. T. Record, P. L. deHaseth and T. M. Lohman, Biochem. J., 1981, 16, 4791.

249 C. G. Kalodimos, et al., Science, 2004, 305, 386.

250 K. Nadassy, S. J. Wodak and J. Janin, Biochemistry, 1999, 38, 1999.

251 P. H. von Hippel and O. G. Berg, Proc. Natl. Acad. Sci. U. S. A., 1986, 83, 1608.

252 N. M. Luscombe, et al., Nucleic Acids Res., 2001, 29, 2860.

253 A. G. Cherstvy, A. A. Kolomeisky and A. A. Kornyshev, J. Phys. Chem. B, 2008, 112, 4741.

254 A. Wynveen, unpublished results.

255 A. N. Temiz, et al., Nucleic Acids Res., 2010, 38, 2134.

256 D. Rajamani, et al., Proc. Natl. Acad. Sci. U. S. A., 2004, 101, 11287.

257 A. G. Cherstvy, J. Phys. Chem. B, 2009, 113, 4242.

258 P. A. Rice, et al., Cell, 1996, 87, 1295.

259 V. B. Teif and K. Rippe, Nucleic Acids Res., 2009, 37, 5641.

260 E. Segal, et al., Nature, 2006, 442, 772.

261 H. C. Drew and A. A. Travers, J. Mol. Biol., 1985, 186, 773.

262 D. Wang, N. B. Ulyanov and V. B. Zhurkin, J. Biomol. Struct. Dyn., 2010, 27, 843.

263 B. Honig, et al., Nature, 2009, 461, 1248.

264 V. A. Parsegian, Nature, 1969, 221, 844.

265 A. A. Rashin and B. Honig, J. Phys. Chem., 1985, 89, 5588.

266 M. Feig, et al., J. Comput. Chem., 2004, 25, 265.

267 A. G. Cherstvy, J. Phys. Chem. B, 2006, 110, 14503.

268 G. Sigalov, A. Fenley and A. Onufriev, J. Chem. Phys., 2006, 124, 124902 .

269 D. S. Palmer, et al., J. Phys.: Condens. Matter, 2010, 22, 492101.

270 E. L. Ratkova, et al., J. Phys. Chem. B, 2010, 114, 12068.

271 M. V. Fedorov, et al., Phys. Chem. Chem. Phys., 2007, 9, 5423.

272 J. Mongan, et al., J. Chem. Theory Comput., 2007, 3, 156.

273 M. V. Fedorov, et al., J. Am. Chem. Soc., 2009, 131, 10854.

274 T. Takahashi, Adv. Biophys., 1997, 34, 41.

275 M. Vijayakumar, et al., J. Mol. Biol., 1998, 278, 1015.

276 G. Schreiber and A. R. Fersht, Nat. Struct. Biol., 1996, 3, 427.
277 G. Schreiber, G. Haran and H.-X. Zhou, Chem. Rev., 2009, 109, 839.

278 O. Keksin, et al., Chem. Rev., 2008, 108, 1225.

279 S. Kumar and R. Nussinov, ChemBioChem, 2002, 3, 604.

280 O. Keksin, B. Y. Ma and R. Nussinov, J. Mol. Biol., 2005, 245, 1281.

281 C. N. Schutz and A. Warshel, Proteins: Struct., Funct., Bioinf., 2001, 44, 400.

282 D. P. Doerr and Y.-K. Yu, Phys. Rev. E: Stat., Nonlinear, Soft Matter Phys., 2010, 81, 031925.

283 A. T. Fenley, J. C. Gordon and A. Onufriev, J. Chem. Phys., 2008, 129, 075101.

284 J. Antosiewicz, et al., Biochemistry, 1996, 35, 7819.

285 J. Antosiewicz, et al., J. Comput. Chem., 1996, 17, 1633.

286 P. L. Privalov, et al., Nucleic Acids Res., 2011, DOI: 10.1093/ nar/gkq984.

287 R. E. Dickerson, et al., Proc. Natl. Acad. Sci. U. S. A., 1994, 91, 3579.

288 V. K. Misra, et al., Biophys. J., 1998, 75, 2262.

289 T. D. Yager, C. T. McMurray and K. E. van Holde, Biochemistry, 1989, 28, 2271.

290 J. Skolnick and M. Fixman, Macromolecules, 1977, 10, 944.

291 T. J. Odijk, J. Pol. Sci., 1977, 15, 477.

292 R. Everaers, A. Milchev and V. Yamakov, Europ. Phys. J. E, 2002, 83.

293 M. Fixman, J. Phys. Chem. B, 2010, 114, 3185.

294 J.-M. Y. Carrillo and A. V. Dobrynin, Macromolecules, 2010, 43, 2589.

295 V. A. Andreev and A. I. Victorov, J. Chem. Phys., 2010, 132, 054902.

296 A. G. Cherstvy, Eur. Biophys. J., 2011, 40, 69.

297 A. D. Mirzabekov and A. Rich, Proc. Natl. Acad. Sci. U. S. A., 1979, 76, 1118

298 J. K. Strauss and L. J. Maher III, Science, 1994, 266, 1829.

299 A. Yu. Grosberg, T. T. Nguyen and B. I. Shklovskii, Rev. Mod. Phys., 2002, 74, 329.

300 A. T. Fenley, et al., Biophys. J., 2010, 99, 1577.

301 S. V. Lyulin, A. A. Darinskii and A. V. Lyulin, Macromolecules, $2005, \mathbf{3 8}, 3990$.

302 S. V. Larin, et al., J. Phys. Chem. B, 2010, 114, 2910.

303 Y. Higuchi, et al., Phys. Rev. E: Stat., Nonlinear, Soft Matter Phys., 2010, 82, 031909.

304 S. J. de Carvalho, Europhys. Lett., 2010, 92, 18001.

305 K. Qamhieh, T. Nylander and M.-L. Ainalem, Biomacromolecules, 2009, 10, 1720.

306 A. A. Zinchenko, K. Yoshikawa and D. Baigl, Phys. Rev. Lett., 2005, 95, 228101.

307 S. Ulrich, et al., Curr. Opin. Colloid Interface Sci., 2006, 11, 268.

308 L. Arcesi, et al., Biopolymers, 2007, 86, 127.

309 K.-K. Kunze and R. R. Netz, Phys. Rev. Lett., 2000, 85, 4389.

310 A. G. Cherstvy and R. G. Winkler, J. Chem. Phys., 2006, 125, 064904.

311 A. G. Cherstvy and R. G. Winkler, J. Chem. Phys., 2004, 120, 9394.

312 A. G. Cherstvy and R. G. Winkler, J. Phys. Chem. B, 2005, 109, 2962.

313 O. Perisic, et al., J. Mol. Biol., 2010, 403, 777.

314 C. L. Woodcock, A. I. Skoultchi and Y. Fan, Chromosome Res., 2006, 14, 17.

315 A. Routh, et al., Proc. Natl. Acad. Sci. U. S. A., 2008, 105, 8872.

316 K. Luger and T. J. Richmond, Curr. Opin. Genet. Dev., 1998, 8, 140.

317 J. T. Finch and A. Klug, Proc. Natl. Acad. Sci. U. S. A., 1976, 73, 1897.

318 B. Dorigo, et al., Science, 2004, 306, 1571.

319 T. Schalch, et al., Nature, 2005, 436, 138.

320 K. Maeshima, S. Hihara and M. Eltsov, Curr. Opin. Cell Biol., 2010, 22, 291.

321 A. Rosa, N. B. Becker and R. Everaers, Biophys. J., 2010, 98, 2410.

322 K. Finan, P. R. Cook and D. Marenduzzo, Chromosome Res., 2010, 19, 53 .

323 C. C. Fritsch and J. Langowski, Chromosome Res., 2010, 19, 63.

324 E. Lieberman-Aiden, et al., Science, 2009, 326, 289.

325 J. Dekker, et al., Science, 2002, 295, 1306. 
326 K. Nasmyth and C. H. Haering, Annu. Rev. Biochem., 2005, 74, 595.

327 J. J. Griese, et al., Nucleic Acids Res., 2010, 38, 3454.

328 P. J. J. Robinson, et al., Proc. Natl. Acad. Sci. U. S. A., 2006, 103, 6506.

329 K. Luger and T. J. Richmond, Curr. Opin. Struct. Biol., 1998, 8, 33 .

330 S. H. Syed, et al., Proc. Natl. Acad. Sci. U. S. A., 2010, 107, 9620.

331 C. L. White, R. K. Suto and K. Luger, EMBO J., 2001, 20, 5207.

332 Y. Cui and C. Bustamante, Proc. Natl. Acad. Sci. U. S. A., 2000, 97, 127.

333 M. Kruithof, et al., Nat. Struct. Mol. Biol., 2009, 16, 534.

334 A. Bancaud, et al., Nat. Struct. Mol. Biol., 2006, 13, 444.

335 G. Li, et al., Nat. Struct. Mol. Biol., 2004, 15, 46.

336 H. Schiessel, Eur. Phys. J. E, 2006, 19, 371.

337 V. B. Teif, R. Ettig and K. Rippe, Biophys. J., 2010, 99, 2597.

338 V. B Teif and K. Rippe, J. Phys.: Condens. Matter, 2010, 22, 414105 .

339 A. Moebius, R. A. Naher and U. Gerland, Phys. Rev. Lett., 2006, 97, 208102.

340 L. Mollazadeh-Beidokhti, et al., Phys. Rev. E: Stat., Nonlinear, Soft Matter Phys., 2009, 79, 031922.

341 A. Leforestier, J. Dubochet and F. Livolant, Biophys. J., 2001, 81, 2414.

342 J. T. Finch, et al., Nature, 1977, 269, 29.

343 S. Mangenot, et al., J. Mol. Biol., 2003, 333, 907.

344 J. Dubochet and M. Noll, Science, 1978, 202, 280.

345 S. Mangenot, et al., Biophys. J., 2003, 84, 2570.

346 S. Mangenot, et al., Eur. Phys. J. E: Soft Matter Biol. Phys., 2002, $7,221$.

347 F. Mühlbacher, et al., Europhys. Lett., 2006, 73, 135.

348 N. Korolev, et al., Adv. Colloid Interface Sci., 2010, 158, 32.

349 Y. Yang, A. P. Lyubartsev, N. Korolev and L. Nordenskiold, Biophys. J., 2009, 96, 2082.

350 R. Podgornik, T. Ảkesson and B. Jönsson, J. Chem. Phys., 1995, 102, 9423 .

351 S. Mangenot, et al., Biophys. J., 2002, 82, 345.

352 F. Livolant, et al., Philos. Trans. R. Soc. London, Ser. A, 2006, 364, 2615.

353 K. Luger, et al., Proc. Natl. Acad. Sci. U. S. A., 2004, 101, 6864.

354 A. G. Cherstvy and R. Everaers, J. Phys.: Condens. Matter, 2006, 18, 11429.

355 D. Vasudevan, J. Mol. Biol., 2010, 4031.

356 C. A. Davey, et al., J. Mol. Biol., 2002, 319, 1097.

357 Y. Tsunaka, et al., Nucleic Acids Res., 2005, 33, 3424.

358 A. G. Cherstvy, et al., work in preparation.

359 V. Dahirel, et al., J. Chem. Phys., 2007, 127, 095101.

360 M. Fixman and J. Skolnick, Macromolecules, 1978, 11, 863.

361 A. G. Cherstvy, J. Phys. Chem. B, 2007, 111, 12933.

362 Z. Zhang and S. C. Glotzer, Nano Lett., 2004, 4, 1407.

363 M. Depken and H. Schiessel, Biophys. J., 2009, 96, 777.

364 B. Mergell, R. Everaers and H. Schiessel, Phys. Rev. E: Stat. Nonlinear, Soft Matter Phys., 2004, 70, 011915.

365 H. Schiessel, W. M. Gelbart and R. Bruinsma, Biophys. J., 2001, 80, 1940

366 G. Wedemann and J. Langowski, Biophys. J., 2002, 82, 2847.

367 J. Sun, Q. Zhang and T. Schlick, Proc. Natl. Acad. Sci. U. S. A., $2005, \mathbf{1 0 2}, 8180$.

368 R. Stehr, et al., Biophys. J., 2008, 95, 3677.

369 P. M. Diesinger and D. W. Heermann, Biophys. J., 2009, 97, 2146

370 N. Korolev, et al., Biophys. J., 2010, 99, 1896.

371 W. C. Earnshaw and S. C. Harrison, Nature, 1977, 268, 598.

372 W. M. Gelbart and C. M. Knobler, Phys. Today, 2008, 61, 42.

373 W. H. Roos, et al., Cell. Mol. Life Sci., 2007, 64, 1484.

374 W. M. Gelbart and C. M. Knobler, Science, 2009, 323, 1682.

375 D. E. Smith, et al., Nature, 2001, 413, 748.

376 D. N. Fuller, et al., Proc. Natl. Acad. Sci. U. S. A., 2007, 104, 11245.

377 P. Grayson, et al., Proc. Natl. Acad. Sci. U. S. A., 2007, 104, 14652.

378 T. S. Baker, et al., Microbiol. Mol. Biol. Rev., 1999, 63, 862.

379 V. S. Reddy, et al., J. Virol., 2001, 75, 11943.

$380 \mathrm{http}: / /$ viperdb.scripps.edu.

381 www.rcsb.org.

382 M. E. Cerritelli, et al., Cell, 1997, 91, 271.
383 A. Fokine, et al., Proc. Natl. Acad. Sci. U. S. A., 2004, 101, 6003. 384 N. H. Olson, et al., Virology, 2001, 279, 385.

385 J. Johnson, et al., Science, 2006, 312, 1791.

386 A. Leforestier and F. Livolant, Proc. Natl. Acad. Sci. U. S. A., 2009, 106, 9157.

387 A. Leforestier and F. Livolant, J. Mol. Biol., 2010, 396, 384.

388 A. Evilevitch, et al., Biophys. J., 2008, 94, 1110.

389 A. Evilevitch, et al., J. Phys. Chem. B, 2004, 108, 6838.

390 R. Metzler and P. G. Dommersnes, Eur. Biophys. J., 2004, 33, 497.

391 P. K. Purohit, J. Kondev and R. Phillips, Proc. Natl. Acad. Sci. U. S. A., 2003, 100, 3173.

392 P. K. Purohit, et al., Biophys. J., 2005, 88, 851.

393 M. Castelnovo and A. Evilevitch, Eur. Phys. J. C, 2007, 24, 9.

394 D. G. Angelescu, et al., Phys. Rev. E: Stat., Nonlinear, Soft Matter Phys., 2006, 73, 041921.

395 D. G. Angelescu, et al., J. Phys. Chem. B, 2007, 111, 8477.

396 Y. Hu, et al., Biophys. J., 2008, 94, 1428.

397 D. Svenšek, G. Veble and R. Podgornik, Phys. Rev. E: Stat., Nonlinear, Soft Matter Phys., 2010, 82, 011708.

398 A. Kivenson and M. F. Hagan, Biophys. J., 2010, 99, 619.

399 D. Marenduzzo, et al., Proc. Natl. Acad. Sci. U. S. A., 2009, 106, 22269.

400 D. Marenduzzo, C. Micheletti and E. Orlandini, J. Phys.. Condens. Matter, 2010, 22, 283102.

401 O. M. Elrad and M. F. Hagan, Phys. Biol., 2010, 7, 045003.

402 D. Eisenberg and A. D. McLachlan, Nature, 1986, 319, 199.

403 P. Ceres and A. Zlotnick, Biochemistry, 2002, 41, 11525.

404 D. M. Salunke, D. L. D. Caspar and R. L. Garsea, Biophys. J., 1989, 56, 887.

405 A. Zlotnick, et al., Biochemistry, 1996, 35, 7412.

406 S. Kanesashi, et al., J. Gen. Virol., 2003, 84, 1899.

407 R. Konechny, et al., Biopolymers, 2004, 75, 325.

408 R. Konechny, et al., Biopolymers, 2006, 82, 106.

409 Y. Miao, et al., J. Phys. Chem. B, 2010, 114, 11181.

410 V. A. Belyi and M. Muthukumar, Proc. Natl. Acad. Sci. U. S. A., 2006, 103, 17174.

411 T. Hu, et al., Phys. A, 2008, 387, 3059.

412 C. Forrey and M. Muthukumar, J. Chem. Phys., 2009, 131, 105101.

413 M. Tihova, et al., J. Virol., 2004, 78, 2897.

414 A. Siber and R. Podgornik, Phys. Rev. E: Stat., Nonlinear, Soft Matter Phys., 2007, 76, 061906.

415 A. Siber and R. Podgornik, Phys. Rev. E: Stat., Nonlinear, Soft Matter Phys., 2008, 78, 051915.

416 M. F. Hagan, J. Chem. Phys., 2009, 130, 114902.

417 A. Siber, et al., Phys. Rev. E: Stat., Nonlinear, Soft Matter Phys., 2010, 81, 051919.

418 D. Svensek, et al., Phys. Rev. E: Stat., Nonlinear, Soft Matter Phys., 2010, 82, 011708.

419 P. Prinsen, et al., J. Phys. Chem. B, 2010, 114, 5522.

420 W. K. Kegel and P. van der Schoot, Biophys. J., 2004, 86, 3905.

421 P. van der Schoot and R. Bruinsma, Phys. Rev. E: Stat., Nonlinear, Soft Matter Phys., 2005, 71, 061928.

422 G. Vernizzi and M. O. de la Cruz, Proc. Natl. Acad. Sci. U. S. A., 2007, 104, 18382.

423 R. Schwartz, et al., Biophys. J., 1998, 75, 2626.

424 D. C. Rapaport, Phys. Rev. E: Stat., Nonlinear, Soft Matter Phys., 2004, 70, 051905.

425 V. S. Reddy, et al., Biophys. J., 1998, 74, 546.

426 S. C. Harvey, et al., Phys. Chem. Chem. Phys., 2009, 11, 10553.

427 W. K. Kegel and P. van der Schoot, Biophys. J., 2006, 91, 1501

428 J. Sun, et al., Proc. Natl. Acad. Sci. U. S. A., 2007, 104, 1354.

429 O. M. Elrad and M. F. Hagan, Nano Lett., 2008, 8, 3850.

430 D. Zhang, et al., Biopolymers, 2004, 75, 325.

431 S. Kumar and R. Nussinov, ChemBioChem, 2002, 3, 604.

432 W. H. Roos, R. Bruinsma and G. J. L. Wuite, Nat. Phys., 2010, 6, 733.

433 J. Lidmar, L. Mirny and D. R. Nelson, Phys. Rev. E: Stat. Phys., Plasmas, Fluids, Relat. Interdiscip. Top., 2003, 68, 051910.

434 T. T. Nguyen, et al., Phys. Rev. Lett., 2006, 96, 078102.

435 V. L. Lorman and S. B. Rochal, Phys. Rev. Lett., 2007, 98, 185502.

436 T. T. Nguyen, R. F. Bruinsma and W. M. Gelbart, Phys. Rev. E: Stat., Nonlinear, Soft Matter Phys., 2005, 72, 051923. 
437 M. Buenemann and P. Lenz, Proc. Natl. Acad. Sci. U. S. A., 2007, 104, 9925.

438 G. A. Vliegenthart and G. Gompper, Biophys. J., 2006, 91, 834.

439 R. Zandi and D. Reguera, Phys. Rev. E: Stat., Nonlinear, Soft Matter Phys., 2005, 72, 021917.

440 W. S. Klug, et al., Phys. Rev. Lett., 2006, 97, 228101.

441 M. Baclayon, G. J. L. Wuite and W. H. Roos, Soft Matter, 2010, 6, 5273.

442 I. L. Ivanovska, et al., Proc. Natl. Acad. Sci. U. S. A., 2004, 101, 7600.

443 D. L. D. Caspar and A. Klug, Cold Spring Harbor Symp., 1962, $27,1$.

444 I. L. Ivanovska, et al., Proc. Natl. Acad. Sci. U. S. A., 2007, 104, 9603.

445 A Evilevitch, J. Mol. Biol., 2011, 405, 18.

446 J. P. Michel, et al., Proc. Natl. Acad. Sci. U. S. A., 2006, 103, 6184.

447 S. Mangenot, M. Hochrein, J. Rädler and L. Letellier, Curr. Biol., $2005, \mathbf{1 5}, 430$.

448 M. M. Inamdar, W. M. Gelbart and R. Phillips, Biophys. J., 2006, 91, 411 .
449 C. Wong and M. Muthukumar, J. Chem. Phys., 2010, 133, 045101.

450 W. H. Roos and G. J. L. Wuite, Adv. Mater., 2009, 21, 1187.

451 A. G. Cherstvy and R. G. Winkler, Phys. Rev. Lett., 2006, 96, 066103.

452 R. G. Winkler and A. G. Cherstvy, J. Phys. Chem. B, 2007, 111, 8486.

453 A. G. Cherstvy, J. Phys. Chem. B, 2011, submitted.

454 M. Muthukumar, J. Chem. Phys., 1995, 103, 4723.

455 N. Hoda and S. Kumar, J. Chem. Phys., 2008, 128, 124907.

456 A. Zlotnick, personal communication.

457 A. Arkhipov, et al., Biophys. J., 2009, 97, 2061.

458 C. Carrasco, et al., Proc. Natl. Acad. Sci. U. S. A., 2006, 103, 13706.

459 W. H. Roos, et al., Biophys. J., 2010, 99, 1175.

460 M. M. Gibbons and W. S. Klug, Biophys. J., 2008, 95, 3640.

461 C. Carrasco, et al., Proc. Natl. Acad. Sci. U. S. A., 2008, 105, 4150.

462 R. Mateo, et al., J. Biol. Chem., 2003, 278, 41019.

463 R. Mateo, et al., J. Virol., 2008, 82, 12232.

464 M. G. Mateo, Protein Eng., Des. Sel., 2011, 24, 53. 